TRANSACTIONS OF THE

AMERICAN MATHEMATICAL SOCIETY

Volume 365, Number 3, March 2013, Pages 1149-1181

S 0002-9947(2012)05550-4

Article electronically published on October 2, 2012

\title{
THE GENUS ONE GROMOV-WITTEN INVARIANTS OF CALABI-YAU COMPLETE INTERSECTIONS
}

\author{
ALEXANDRA POPA
}

\begin{abstract}
We obtain mirror formulas for the genus 1 Gromov-Witten invariants of projective Calabi-Yau complete intersections. We follow the approach previously used for projective hypersurfaces by extending the scope of its algebraic results; there is little change in the geometric aspects. As an application, we check the genus 1 BPS integrality predictions in low degrees for all projective complete intersections of dimensions 3,4 , and 5 .
\end{abstract}

\section{Contents}

1. Mirror symmetry formulas

1149

1.1. Statement of main theorem

1149

1.2. Special cases of main theorem

1152

2. Outline of the proof

1155

3. Equivariant setup

1158

3.1. Equivariant cohomology

1158

3.2. Generating function for reduced genus $1 \mathrm{GW}$ invariants $\quad 1160$

3.3. A localization proposition

4. Some properties of hypergeometric series $\tilde{\mathcal{F}}$

4.1. Proof of Lemma 4.1 and Proposition 4.4

4.2. Proof of equation 4.5.

4.3. Proof of equation (4.6)

5. Computation of reduced genus $1 \mathrm{GW}$ invariants 1171

5.1. Some algebraic notation and observations 1172

5.2. The genus 0 generating functions 1174

5.3. Proof of Theorem 3

References

\section{MirRor SYMmetry FORMULAS}

1.1. Statement of main theorem. Gromov-Witten invariants of projective varieties are counts of curves that are conjectured (and known in some cases) to possess a rich structure. The original mirror prediction of CaDGP for the genus 0 Gromov-Witten invariants of a quintic threefold has since been verified and shown to be a special case of mirror formulas satisfied by Gromov-Witten invariants of

Received by the editors October 15, 2010 and, in revised form, January 26, 2011.

2010 Mathematics Subject Classification. Primary 14N35.

This research was partially supported by DMS grant 0846978 .

(C)2012 American Mathematical Society Reverts to public domain 28 years from publication 
complete intersections; see [Gi] and [LLY]. Mirror formulas for the genus 1 GromovWitten invariants of projective Calabi-Yau hypersurfaces are obtained in [Z4] and [Z5], in particular confirming the prediction of [BCOV] for a quintic threefold. In this paper, we obtain mirror formulas for the genus 1 Gromov-Witten invariants of all projective Calabi-Yau complete intersections following the approach in [Z5, extending $[\mathrm{ZaZ}$, and using $[\mathrm{PoZ}]$ in place of [Z3].

Let $X_{\mathbf{a}}$ be a smooth complete intersection of $l$ hypersurfaces in $\mathbb{P}^{n-1}$ of degrees $a_{1}, a_{2}, \ldots, a_{l} \in \mathbb{Z}^{>0}$, where

$$
\mathbf{a} \equiv\left(a_{1}, a_{2}, \ldots, a_{l}\right)
$$

This complete intersection is Calabi-Yau if and only if $\sum_{r=1}^{l} a_{r}=n$; from now on it will be assumed that this condition holds. Let $N_{1}^{d}\left(X_{\mathbf{a}}\right) \in \mathbb{Q}$ denote the degree $d$ genus 1 Gromov-Witten invariant of $X_{\mathbf{a}}$. As often done in Gromov-Witten theory, we assemble these numbers into a generating series,

$$
\sum_{d=1}^{\infty} N_{1}^{d}\left(X_{\mathbf{a}}\right) Q^{d} \in Q \cdot \mathbb{Q}[[Q]]
$$

Theorem 1 relates this formal power series to an explicit hypergeometric series and thus determines all numbers $N_{1}^{d}\left(X_{\mathbf{a}}\right)$.

As in the genus 0 case treated in [Gi] and [LLY], the hypergeometric series encoding the genus 1 Gromov-Witten invariants of $X_{\mathbf{a}}$ is

$$
\tilde{\mathcal{F}}(w, q) \equiv \sum_{d=0}^{\infty} q^{d} \frac{\prod_{k=1}^{l} \prod_{r=1}^{a_{k} d}\left(a_{k} w+r\right)}{\prod_{r=1}^{d}(w+r)^{n}} \in \mathbb{Q}(w)[[q]] .
$$

This is a power series in $q$ with constant term 1 whose coefficients are rational functions in $w$ which are holomorphic at $w=0$. As in [ZaZ], we denote by

$$
\mathcal{P} \subset 1+q \mathbb{Q}(w)[[q]]
$$

the subgroup of all such power series. Thus, the evaluation map

$$
\mathcal{P} \rightarrow 1+q \mathbb{Q}[[q]], \quad F(w, q) \longrightarrow F(0, q),
$$

is well defined. We will also need transforms of $\tilde{\mathcal{F}}(w, q)$ under the map

$$
\mathbf{M}: \mathcal{P} \rightarrow \mathcal{P}, \quad \mathbf{M} F(w, q) \equiv\left\{1+\frac{q}{w} \frac{\mathrm{d}}{\mathrm{d} q}\right\} \frac{F(w, q)}{F(0, q)}
$$

For $p=0,1, \ldots, n-1$, set

$$
I_{p}(q) \equiv \mathbf{M}^{p} \tilde{\mathcal{F}}(0, q) \in 1+q \cdot \mathbb{Q}[[q]] .
$$


For example,

$$
I_{0}(q)=\sum_{d=0}^{\infty} q^{d} \frac{\left(a_{1} d\right) !\left(a_{2} d\right) ! \ldots\left(a_{l} d\right) !}{(d !)^{n}} .
$$

In Theorem 1, the formal variables $Q$ in (1.1) and $q$ in (1.2) are related by the usual mirror map

$$
Q=q \mathrm{e}^{J(q)},
$$

where

$$
\left.J(q) \equiv \frac{1}{I_{0}(q)} \frac{\mathrm{d} \tilde{\mathcal{F}}(w, q)}{\mathrm{d} w}\right|_{w=0}=\frac{1}{I_{0}(q)}\left\{\sum_{d=1}^{\infty} q^{d} \frac{\prod_{k=1}^{l}\left(a_{k} d\right) !}{(d !)^{n}}\left(\sum_{k=1}^{l} \sum_{r=d+1}^{a_{k} d} \frac{a_{k}}{r}\right)\right\} .
$$

The power series $J(q)$ can also be defined by

$$
J(q) \in q \cdot \mathbb{Q}[[q]], \quad q \frac{\mathrm{d} J(q)}{\mathrm{d} q}=I_{1}(q)-1 .
$$

The map $q \longrightarrow Q$ is a change of formal variables, in the sense that it induces an isomorphism

$$
q \cdot \mathbb{Q}[[q]] \longrightarrow Q \cdot \mathbb{Q}[[Q]]
$$

of the vector spaces of power series in the two variables with no constant term.

Finally, we define $\langle\mathbf{a}\rangle, \mathbf{a}^{\mathbf{a}}, \varepsilon_{0}(\mathbf{a}), \varepsilon_{1}(\mathbf{a}) \in \mathbb{Z}$ by

$$
\langle\mathbf{a}\rangle \equiv \prod_{k=1}^{l} a_{k}, \quad \mathbf{a}^{\mathbf{a}} \equiv \prod_{k=1}^{l} a_{k}^{a_{k}},\left.\quad c_{n-1-l-i}\left(X_{\mathbf{a}}\right) \equiv \varepsilon_{i}(\mathbf{a}) \mathrm{H}^{n-1-l-i}\right|_{X_{\mathbf{a}}},
$$

where $\mathrm{H} \in H^{2}\left(\mathbb{P}^{n-1}\right)$ is the hyperplane class (set $\varepsilon_{1}(\mathbf{a})=0$ if $l=n-1, n$ and $\varepsilon_{0}(\mathbf{a})=0$ if $\left.l=n\right)$.

Theorem 1. The genus 1 Gromov-Witten invariants of a multi-degree a CalabiYau complete intersection $X_{\mathbf{a}}$ in $\mathbb{P}^{n-1}$ are given by

$$
\begin{aligned}
& \sum_{d=1}^{\infty} N_{1}^{d}\left(X_{\mathbf{a}}\right) Q^{d}=\frac{\langle\mathbf{a}\rangle}{24} \varepsilon_{0}(\mathbf{a})\left[\log I_{0}(q)\right]+\frac{\langle\mathbf{a}\rangle}{24} \varepsilon_{1}(\mathbf{a}) J(q) \\
& - \begin{cases}\frac{n-l}{48} \log \left(1-\mathbf{a}^{\mathbf{a}} q\right)+\frac{\sum_{p=0}^{\frac{n-2-l}{2}} \frac{(n-l-2 p)^{2}}{8}}{p} \log I_{p}(q), & \text { if } 2 \mid(n-l), \\
\frac{n-3-l}{48} \log \left(1-\mathbf{a}^{\mathbf{a}} q\right)+\frac{\sum_{p=0}^{\frac{n-3-l}{2}}}{\sum_{p}} \frac{(n-l-2 p)^{2}-1}{8} \log I_{p}(q), & \text { if } 2 \nmid(n-l),\end{cases}
\end{aligned}
$$

where $Q \equiv q \mathrm{e}^{J(q)}$.

Note that dropping a component of a equal to 1 does not change the algebraic variety $X_{\mathbf{a}}$, the numbers $\langle\mathbf{a}\rangle, \mathbf{a}^{\mathbf{a}}, \varepsilon_{i}(\mathbf{a})$, or the power series $\tilde{\mathcal{F}}$ in (1.2). It follows that the two sides of the identity in Theorem 1 are a priori invariant under dropping a component of a equal to 1 . Thus, it is sufficient to prove Theorem 1 under the assumption that $a_{k} \geq 2$ for all $k$. We make this assumption throughout the proof, which is outlined in Section $2^{1}$

\footnotetext{
${ }^{1}$ The assumption that $a_{k} \geq 2$ is used to streamline the computations in Section 5 only.
} 
The proof of Theorem 1 relies on the desingularization of the moduli space of genus 1 maps to $\mathbb{P}^{n-1}$ constructed in $[\mathrm{VaZ}$ and the hyperplane relation for reduced genus 1 Gromov-Witten invariants obtained in [LiZ. These results would need to be suitably extended in order to apply the approach of [Z5] to complete intersections in smooth toric varieties other than projective spaces. In the Fano cases, at least one marked point should be added, because the relevant moduli spaces are then (usually) positive dimensional so that constraints must be imposed to get GromovWitten invariants. This would require mirror formulas for genus 0 Gromov-Witten invariants with at least three marked points. Such formulas have recently been obtained by A. Zinger in [Z6]. It should now be possible to extend Theorem 1 to Fano projective complete intersections.

The author would like to express deep gratitude to A. Zinger for explaining [Z4] and [Z5], for proposing the questions answered in this paper, and for his invaluable suggestions. The author is also grateful to the referees for their comments on the initial version of this paper and their suggestions on improving the exposition.

1.2. Special cases of main theorem. In this section we show that the $\mathbf{a}=(n)$ case of Theorem[1 (when $X_{\mathbf{a}}$ is a Calabi-Yau hypersurface) recovers [Z4, Theorem 2]. We then consider low-dimensional cases of Theorem 1, concluding with tables of so-called BPS numbers.

By the adjunction formula, the total Chern class of $X_{\mathrm{a}}$ is given by

$$
c\left(X_{\mathbf{a}}\right)=\frac{\left(1+\left.\mathrm{H}\right|_{X_{\mathbf{a}}}\right)^{n}}{\prod_{r=1}^{l}\left(1+\left.a_{r} \mathrm{H}\right|_{X_{\mathbf{a}}}\right)} \in H^{*}\left(X_{\mathbf{a}}\right) .
$$

Thus, $\varepsilon_{0}(\mathbf{a})$ and $\varepsilon_{1}(\mathbf{a})$ are the coefficients of $w^{n-1-l}$ and $w^{n-2-l}$, respectively, in the power series expansion of

$$
\frac{(1+w)^{n}}{\prod_{r=1}^{l}\left(1+a_{r} w\right)} \in \mathbb{Q}(w)
$$

around $w=0$. If $\mathbf{a}=(n)$, by the Residue Theorem on $S^{2}$,

$$
\begin{aligned}
\varepsilon_{0}(\mathbf{a}) & =\mathfrak{R}_{w=0}\left\{\frac{(1+w)^{n}}{w^{n-1}(1+n w)}\right\} \\
& =-\mathfrak{R}_{w=-1 / n}\left\{\frac{(1+w)^{n}}{w^{n-1}(1+n w)}\right\}+\mathfrak{R}_{z=0}\left\{\frac{(z+1)^{n}}{z^{2}(z+n)}\right\} \\
& =\frac{(n-1)^{n}}{(-1)^{n} n^{2}}+1-\frac{1}{n^{2}}=\frac{n^{2}-1+(1-n)^{n}}{n^{2}}, \\
\varepsilon_{1}(\mathbf{a}) & =\mathfrak{R}_{w=0}\left\{\frac{(1+w)^{n}}{w^{n-2}(1+n w)}\right\} \\
& =-\mathfrak{R}_{w=-1 / n}\left\{\frac{(1+w)^{n}}{w^{n-2}(1+n w)}\right\}+\mathfrak{R}_{z=0}\left\{\frac{(z+1)^{n}}{z^{3}(z+n)}\right\} \\
& =-\frac{(n-1)^{n}}{(-1)^{n} n^{3}}+\frac{n-1}{2}-\frac{1}{n}+\frac{1}{n^{3}}=\frac{(n-2)(n+1)}{2 n}+\frac{1-(1-n)^{n}}{n^{3}},
\end{aligned}
$$

where $\mathfrak{R}_{w=w_{0}}$ denotes the residue at $w=w_{0}$. Thus, the $l=1$ case of Theorem 1 indeed reduces to [Z4, Theorem 2]. 
The cases $l=n\left(X_{\mathbf{a}}=\emptyset\right)$ and $l=n-1\left(X_{\mathbf{a}}\right.$ is $\langle\mathbf{a}\rangle=2$ points $)$ reduce to the case $l=1$; as explained in [Z5, Section 0.3] the right-hand side of the formula in Theorem 1 vanishes as expected.

If $l=n-2, X_{\mathbf{a}}$ is a torus, either $X_{3} \subset \mathbb{P}^{2}$ or $X_{2,2} \subset \mathbb{P}^{3}$. In this case, $N_{1}^{d}\left(X_{\mathbf{a}}\right)$ is the number of degree $d$ maps from genus 1 curves to $X_{\mathbf{a}}$ modulo automorphisms of such maps; see [KlPa, 0.2]. Since any such map is an unramified cover of $X_{\mathbf{a}}$ by a torus, it follows that $N_{1}^{d}\left(X_{2,2}\right)$ is 0 unless $d$ is divisible by 4 and $N_{1}^{4 r}\left(X_{2,2}\right)$ is the number of degree $r$ covers of $X_{2,2}$ by a torus divided by $r$. Thus, using the formula [Z5, (B.12)] for the number of degree $r$ unramified covers of a torus, we obtain

$$
\sum_{d=1}^{\infty} N_{1}^{d}\left(X_{2,2}\right) Q^{d}=-\sum_{r=1}^{\infty} \log \left(1-Q^{4 r}\right) .
$$

This identity together with Theorem 1 implies that

$$
\frac{1}{6} J(q)-\frac{1}{24} \log (1-16 q)-\frac{1}{2} \log I_{0}(q)=-\sum_{r=1}^{\infty} \log \left(1-Q^{4 r}\right) .
$$

The same argument is applied to $X_{3}$ in [Z5, Section 0.3] to obtain

$$
\frac{1}{8} J(q)-\frac{1}{24} \log (1-27 q)-\frac{1}{2} \log I_{0}(q)=-\sum_{r=1}^{\infty} \log \left(1-Q^{3 r}\right) .
$$

The latter identity is verified directly in $\underline{\mathrm{Sc}}$; we expect that similar modular-forms techniques can be used to verify the former identity directly as well.

If $l=n-3, X_{\mathbf{a}}$ is a $\mathrm{K} 3$ surface, either $X_{4} \subset \mathbb{P}^{3}, X_{2,3} \subset \mathbb{P}^{4}$, or $X_{2,2,2} \subset \mathbb{P}^{5}$. Since

$$
\langle\mathbf{a}\rangle \varepsilon_{0}(\mathbf{a})=\chi\left(X_{\mathbf{a}}\right)=24 \quad \text { and } \quad \varepsilon_{1}(\mathbf{a})=0
$$

by (1.8), the right-hand side of the formula in Theorem 1 is 0 in all three cases, as expected (all Gromov-Witten invariants of K3 surfaces vanish).

If $l=n-4, X_{\mathbf{a}} \subset \mathbb{P}^{n-1}$ is a Calabi-Yau threefold. Since Calabi-Yau threefolds are of a particular interest in Gromov-Witten theory, we restate the $l=n-4$ case of Theorem 1 as a corollary below. In this case,

$$
\begin{aligned}
\varepsilon_{0}(\mathbf{a})= & \mathfrak{R}_{w=0}\left\{\frac{(1+w)^{n}}{w^{4} \prod_{r=1}^{l}\left(1+a_{r} w\right)}\right\} \\
= & \frac{n(n-1)(n-2)}{6}-\frac{n(n-1)}{2} \sum_{r=1}^{l} a_{r} \\
& +n \sum_{r_{1}=1}^{l} \sum_{r_{2}=r_{1}}^{l} a_{r_{1}} a_{r_{2}}-\sum_{r_{1}=1}^{l} \sum_{r_{2}=r_{1}}^{l} \sum_{r_{3}=r_{2}}^{l} a_{r_{1}} a_{r_{2}} a_{r_{3}}, \\
\varepsilon_{1}(\mathbf{a})= & \mathfrak{R}_{w=0}\left\{\frac{(1+w)^{n}}{w^{3} \prod_{r=1}^{l}\left(1+a_{r} w\right)}\right\}=\frac{n(n-1)}{2}-n \sum_{r=1}^{l} a_{r}+\sum_{r_{1}=1}^{l} \sum_{r_{2}=r_{1}}^{l} a_{r_{1}} a_{r_{2}} .
\end{aligned}
$$


TABLE 1. Low-degree genus 1 BPS numbers for all CY CI 3-folds

\begin{tabular}{c||c|c|c|c|c}
\hline $\mathrm{d}$ & 3 & 4 & 5 & 6 & 7 \\
\hline$X_{5}$ & 609250 & 3721431625 & 12129909700200 & 31147299732677250 & 71578406022880761750 \\
$X_{24}$ & 2560 & 17407072 & 24834612736 & 23689021707008 & 19078577926517760 \\
$X_{33}$ & 3402 & 5520393 & 4820744484 & 3163476678678 & 1798399482469092 \\
$X_{223}$ & 64 & 265113 & 198087264 & 89191834992 & 32343228035424 \\
$X_{2222}$ & 0 & 14752 & 8782848 & 2672004608 & 615920502784 \\
\hline
\end{tabular}

TABLE 2. Low-degree genus 1 BPS numbers for all CY CI 4-folds

\begin{tabular}{c||c|c|c|c}
\hline $\mathrm{d}$ & 3 & 4 & 5 & 6 \\
\hline$X_{6}$ & 2734099200 & 387176346729900 & 26873294164654597632 & 1418722120880095142462400 \\
$X_{25}$ & 9058000 & 845495712250 & 20201716419250520 & 320471504960631822000 \\
$X_{34}$ & 2813440 & 81906297984 & 1006848150400512 & 8707175700941649792 \\
$X_{224}$ & 47104 & 4277292544 & 42843921424384 & 249771462364601344 \\
$X_{233}$ & 53928 & 1203128235 & 7776816583356 & 31624897877254152 \\
$X_{2223}$ & 1024 & 65526084 & 338199639552 & 923753814135936 \\
$X_{22222}$ & 0 & 3779200 & 15090827264 & 27474707200000 \\
\hline
\end{tabular}

TABLE 3. Low-degree genus 1 BPS numbers for all CY CI 5-folds

\begin{tabular}{c||c|c|c}
\hline $\mathrm{d}$ & 3 & 4 & 5 \\
\hline$X_{7}$ & 26123172457235 & 81545482364153841075 & 117498479295762788677099464 \\
$X_{26}$ & 69072837120 & 101190144588682320 & 41238110240372421024768 \\
$X_{35}$ & 8659735175 & 4075445624973975 & 725876976084810684840 \\
$X_{44}$ & 3950411776 & 1453445296487936 & 201129967921550639104 \\
$X_{225}$ & 254083200 & 244005174397575 & 33504170048610349120 \\
$X_{234}$ & 76664320 & 22674781508976 & 1639705524423750144 \\
$X_{333}$ & 39550437 & 5866761130074 & 289435387120696044 \\
$X_{2224}$ & 1507328 & 1349735463168 & 75612640683245568 \\
$X_{2233}$ & 1532160 & 357068201643 & 13410965796358752 \\
$X_{2223}$ & 32768 & 21650838624 & 622096658307072 \\
$X_{222222}$ & 0 & 1342995456 & 29080932827136 \\
\hline \multicolumn{2}{c}{}
\end{tabular}

\begin{tabular}{c||c}
\hline $\mathrm{d}$ & 6 \\
\hline$X_{7}$ & 126043741686161819224278666855602 \\
$X_{26}$ & 11147640321191212498287799296 \\
$X_{35}$ & 88498079911311785027601450 \\
$X_{44}$ & 19073323868063994075791360 \\
$X_{225}$ & 2706605385511145151653200 \\
$X_{234}$ & 72802469333317263218688 \\
$X_{333}$ & 9086367064035583738332 \\
$X_{2224}$ & 2228706944980098304000 \\
$X_{2233}$ & 278702674357074092928 \\
$X_{22223}$ & 8565078595779227136 \\
$X_{222222}$ & 264415120930570240 \\
\hline
\end{tabular}


Corollary 2. The genus 1 Gromov-Witten invariants of a Calabi-Yau complete intersection threefold $X_{\mathbf{a}} \subset \mathbb{P}^{n-1}$ are given by

$$
\begin{aligned}
\sum_{d=1}^{\infty} N_{1}^{d}\left(X_{\mathbf{a}}\right) Q^{d}= & {\left[-2+\frac{\langle\mathbf{a}\rangle}{72}\left(n-S_{3}(\mathbf{a})\right)\right] \log I_{0}(q)+\frac{\langle\mathbf{a}\rangle}{48}\left(S_{2}(\mathbf{a})-n\right) J(q) } \\
& +\log \left[I_{1}(q)^{-\frac{1}{2}}\left(1-\mathbf{a}^{\mathbf{a}} q\right)^{-\frac{1}{12}}\right]
\end{aligned}
$$

where $S_{p}(\mathbf{a}) \equiv \sum_{r=1}^{l} a_{r}^{p}$ and $Q=q \mathrm{e}^{J(q)}$.

Tables 13 show low-degree genus 1 BPS numbers for all Calabi-Yau complete intersection 3-, 4-, and 5-folds obtained from Theorem 11 using MirSym, (34.3)], KlPa, (3)], and $[\mathrm{PaZ},(0.5)]$, respectively 2 Using computer programs 3 we verified the predicted integrality of these numbers up to degree 100 for all Calabi-Yau complete intersection 3-, 4-, and 5-folds. While the degree 1 and 2 genus 1 BPS numbers are 0 as expected, the degree 3 BPS numbers match the classical Schubert calculus on $G(3, n)$. It should be possible to obtain the degree 4 numbers using the approach of [ESt], which provides such numbers for hypersurfaces.

\section{OUtLine OF THE PROOF}

We prove Theorem 1 following the approach used to prove [Z4, Theorem 2]. Theorem 3, stated at the beginning of Section 5 , computes the reduced genus 1 GW invariants $N_{1}^{d ; 0}\left(X_{\mathbf{a}}\right)$ of $X_{\mathbf{a}}$ defined in [Z1]. These invariants are related to the standard genus 1 invariants by Lemma 2.1, which extends [Z4, Lemma 2.2] to complete intersections and is proved in this section. Theorem 1 follows immediately from Lemma 2.1] (3.9), and Theorem 3

The genus 1 hyperplane theorem of [LiZ] and the desingularization construction of [VaZ express the reduced genus $1 \mathrm{GW}$ invariants of $X_{\mathbf{a}}$ in terms of integrals over smooth spaces of maps to $\mathbb{P}^{n-1}$. We use this in Section 3.2 to package the numbers $N_{1}^{d ; 0}\left(X_{\mathbf{a}}\right)$ into a power series $\mathfrak{X}(\alpha, x, Q)$, in a formal variable $Q$ and with coefficients in the equivariant cohomology of $\mathbb{P}^{n-1}$. As $\mathfrak{X}(\alpha, x, Q)$ involves integrals on smooth moduli spaces, the Atiyah-Bott localization theorem $[\mathrm{ABO}$ can be applied as in [Z5. This leads to Proposition 3.1 of Section 3.3, the latter expresses $\mathfrak{X}(\alpha, x, Q)$ in terms of residues of some genus 0 generating functions.

We extract the "nonequivariant part" of $\mathfrak{X}(\alpha, x, Q)$ in Section [5] using [Z5, Lemma 3.3] and mirror formulas for genus 0 generating functions. This reduces the problem of computing the numbers $N_{1}^{d ; 0}\left(X_{\mathbf{a}}\right)$ to purely algebraic questions concerning the power series (1.2). These are addressed in Section 4, which significantly extends $\mathrm{ZaZ}$; this section can be read independently of the rest of the paper.

All cohomology groups in this paper will be with rational coefficients. We will denote by $[n]$, whenever $n \in \mathbb{Z}^{\geq 0}$, the set of positive integers not exceeding $n$ :

$$
[n] \equiv\{1,2, \ldots, n\} .
$$

Whenever $g, d, k$ and $n$ are nonnegative integers and $X$ is a smooth subvariety of $\mathbb{P}^{n-1}, \overline{\mathfrak{M}}_{g, k}(X, d)$ will denote the moduli space of stable degree $d$ maps into $X$

\footnotetext{
${ }^{2}$ Genus 1 BPS counts in higher dimensions are yet to be defined.

${ }^{3}$ Based on Aleksey Zinger's programs for hypersurfaces.
} 
from genus $g$ curves with $k$ marked points and

$$
\mathrm{ev}_{i}: \overline{\mathfrak{M}}_{g, k}\left(\mathbb{P}^{n-1}, d\right) \quad\left[\mathcal{C}, y_{1}, \ldots, y_{k}, u\right] \longrightarrow u\left(y_{i}\right), \quad i=1,2, \ldots, k,
$$

the evaluation map at the $i$-th marked point; see MirSym, Chapter 24]. For each $m \in \mathbb{Z}^{>0}$, define

$$
\begin{gathered}
\overline{\mathfrak{M}}_{(m)}(X, d) \equiv\left\{\left(b_{i}\right)_{i \in[m]} \in \prod_{i=1}^{m} \overline{\mathfrak{M}}_{0,1}\left(X, d_{i}\right): d_{i} \in \mathbb{Z}^{>0},\right. \\
\left.\sum_{i=1}^{m} d_{i}=d, \operatorname{ev}_{1}\left(b_{i}\right)=\operatorname{ev}_{1}\left(b_{i^{\prime}}\right) \forall i, i^{\prime} \in[m]\right\} \\
\operatorname{ev}_{1}: \overline{\mathfrak{M}}_{(m)}(X, d) \longrightarrow X, \quad\left(b_{i}\right)_{i \in[m]} \longrightarrow \operatorname{ev}_{1}\left(b_{i}\right),
\end{gathered}
$$

where $i$ is any element of $[m]$. For each $i \in[m]$, let

$$
\pi_{i}: \overline{\mathfrak{M}}_{(m)}(X, d) \longrightarrow \bigsqcup_{d_{i} \in \mathbb{Z}>0, d_{i} \leq d} \overline{\mathfrak{M}}_{0,1}\left(X, d_{i}\right)
$$

be the projection onto the $i$-th component. If $p \in \mathbb{Z}^{\geq 0}$, we define $\eta_{p} \in H^{2 p}\left(\overline{\mathfrak{M}}_{(m)}(X, d)\right)$ to be the degree $2 p$ term of

$$
\prod_{i=1}^{m} \pi_{i}^{*} \frac{1}{1-\psi_{1}} \in H^{*}\left(\overline{\mathfrak{M}}_{(m)}(X, d)\right)
$$

Thus, $\eta_{p}$ is the sum of all degree $p$ monomials in $\left\{\pi_{i}^{*} \psi_{1}: i \in[m]\right\}$.

The symmetric group on $m$ elements, $S_{m}$, acts on $\overline{\mathfrak{M}}_{(m)}(X, d)$ by permuting the elements of each $m$-tuple of stable maps. Let

$$
\mathcal{Z}_{(m)}(X, d) \equiv \overline{\mathfrak{M}}_{(m)}(X, d) / S_{m} \text { 岁 }
$$

Since the map ev 1 and the cohomology class $\eta_{p}$ on $\overline{\mathfrak{M}}_{(m)}(X, d)$ are $S_{m}$-invariant, they descend to the quotient:

$$
\mathrm{ev}_{1}: \mathcal{Z}_{(m)}(X, d) \longrightarrow X \quad \text { and } \quad \eta_{p} \in H^{2 p}\left(\mathcal{Z}_{(m)}(X, d)\right) .
$$

Let $\mathfrak{U}$ be the universal curve over $\overline{\mathfrak{M}}_{(m)}\left(\mathbb{P}^{n-1}, d\right)$, with structure map $\pi$ and evaluation map ev:

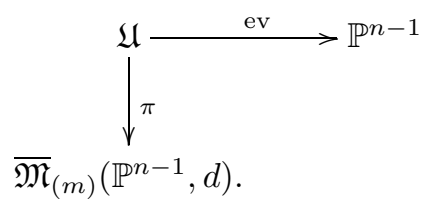

The orbi-sheaf

$$
\pi_{*} \operatorname{ev}^{*} \bigoplus_{r=1}^{l} \mathcal{O}_{\mathbb{P}^{n-1}}\left(a_{r}\right) \longrightarrow \overline{\mathfrak{M}}_{(m)}\left(\mathbb{P}^{n-1}, d\right)
$$

is locally free; it is the sheaf of (holomorphic) sections of the vector orbi-bundle

$$
\mathcal{V}_{(m)} \equiv \overline{\mathfrak{M}}_{(m)}(\mathcal{L}, d) \longrightarrow \overline{\mathfrak{M}}_{(m)}\left(\mathbb{P}^{n-1}, d\right)
$$

\footnotetext{
${ }^{4}$ This space is an orbifold quotient and carries a virtual fundamental class, as explained in $\mathbf{Z} 4$ Section 2.1].
} 
where $\mathcal{L} \longrightarrow \mathbb{P}^{n-1}$ is the total space of the vector bundle corresponding to the sheaf $\bigoplus_{r=1}^{l} \mathcal{O}_{\mathbb{P}^{n-1}}\left(a_{r}\right)$. By the (genus zero) hyperplane-section relation,

$$
\begin{aligned}
& \left\langle\eta_{p-2 m} \mathrm{ev}_{1}^{*} \mathrm{H}^{n-1-l-p},\left[\mathcal{Z}_{(m)}\left(X_{\mathbf{a}}, d\right)\right]^{v i r}\right\rangle \\
& =\frac{1}{m !}\left\langle\eta_{p-2 m} \mathrm{ev}_{1}^{*} \mathrm{H}^{n-1-l-p} e\left(\mathcal{V}_{(m)}\right),\left[\overline{\mathfrak{M}}_{(m)}\left(\mathbb{P}^{n-1}, d\right)\right]\right\rangle,
\end{aligned}
$$

where $\mathrm{H} \in H^{2}\left(\mathbb{P}^{n-1}\right)$ is the hyperplane class.

There is a natural surjective bundle homomorphism

$$
\widetilde{\mathrm{ev}}_{1}: \mathcal{V}_{(1)} \longrightarrow \mathrm{ev}_{1}^{*} \bigoplus_{r=1}^{l} \mathcal{O}_{\mathbb{P}^{n-1}}\left(a_{r}\right), \quad([\mathcal{C}, u, \xi]) \longrightarrow \xi\left(y_{1}(\mathcal{C})\right)
$$

over $\overline{\mathfrak{M}}_{(1)}\left(\mathbb{P}^{n-1}, d\right) \equiv \overline{\mathfrak{M}}_{0,1}\left(\mathbb{P}^{n-1}, d\right)$, where $y_{1}(\mathcal{C})$ is the marked point. Thus,

$$
\mathcal{V}_{(1)}^{\prime} \equiv \operatorname{ker} \widetilde{e v}_{1} \longrightarrow \overline{\mathfrak{M}}_{(1)}\left(\mathbb{P}^{n-1}, d\right)
$$

is a vector orbi-bundle 5 It is straightforward to see that

$$
e\left(\mathcal{V}_{(m)}\right)=\langle\mathbf{a}\rangle \mathrm{ev}_{1}^{*} \mathrm{H}^{l} \prod_{i=1}^{m} \pi_{i}^{*} e\left(\mathcal{V}_{(1)}^{\prime}\right)
$$

If $f=f(w)$ admits a Laurent series expansion around $w=0$, for any $p \in \mathbb{Z}$ we denote by $\llbracket f(w) \rrbracket_{w ; p}$ the coefficient of $w^{p}$. Let

$$
\llbracket \sum_{d=0}^{\infty} f_{d}(w) Q^{d} \rrbracket_{w ; p} \equiv \sum_{d=0}^{\infty} \llbracket f_{d}(w) \rrbracket_{w ; p} Q^{d} \quad \text { if } \quad f_{d} \in \mathbb{Q}(w) \forall d \geq 0 .
$$

Lemma 2.1. If $X_{\mathbf{a}} \subset \mathbb{P}^{n-1}$ is a complete intersection of multidegree $\mathbf{a}$,

$$
\begin{aligned}
& N_{1}^{d}\left(X_{\mathbf{a}}\right)=N_{1}^{d ; 0}\left(X_{\mathbf{a}}\right) \\
&+\frac{1}{24} \sum_{p=2}^{n-1-l} \sum_{m=1}^{2 m \leq p}(-1)^{m}(m-1) ! \\
& \times\left\langle\eta_{p-2 m} \operatorname{ev}_{1}^{*}\left(c_{n-1-l-p}\left(X_{\mathbf{a}}\right)\right),\left[\mathcal{Z}_{(m)}\left(X_{\mathbf{a}}, d\right)\right]^{v i r}\right\rangle .
\end{aligned}
$$

Furthermore, for all $p \in \mathbb{Z}^{\geq 0}$

$$
c_{p}\left(X_{\mathbf{a}}\right)=\llbracket \frac{(1+w)^{n}}{\prod_{r=1}^{l}\left(1+a_{r} w\right)} \|\left._{w ; p} \mathrm{H}^{p}\right|_{X_{\mathbf{a}}},
$$

and for all $p$ with $2 \leq p \leq n-1-l$

$$
\begin{aligned}
\sum_{d=1}^{\infty} Q^{d} & \left(\sum_{m=1}^{2 m \leq p}(-1)^{m}(m-1) !\left\langle\left.\eta_{p-2 m} \mathrm{ev}_{1}^{*} \mathrm{H}^{n-1-l-p}\right|_{X_{\mathbf{a}}},\left[\mathcal{Z}_{(m)}\left(X_{\mathbf{a}}, d\right)\right]^{v i r}\right\rangle\right) \\
= & -\langle\mathbf{a}\rangle \llbracket \log \frac{\tilde{\mathcal{F}}(w, q)}{I_{0}(q)} \rrbracket_{w ; p},
\end{aligned}
$$

where $\mathrm{H} \in H^{2}\left(\mathbb{P}^{n-1}\right)$ is the hyperplane class, $Q$ and $q$ are related by the mirror map (1.6), and $\tilde{\mathcal{F}}(w, q)$ and $I_{0}(q)$ are given by (1.2) and (1.5), respectively.

\footnotetext{
${ }^{5}$ In the notation of Section $3.2 \mathcal{V}_{(1)}=\mathcal{V}_{0}$ and $\mathcal{V}_{(1)}^{\prime}=\mathcal{V}_{0}^{\prime}$
} 
Proof. The first identity above is a special case of [Z4, (2.15)]. The second identity is immediate from

$$
c\left(T \mathbb{P}^{n-1}\right)=(1+\mathrm{H})^{n} \quad \text { and } \quad c\left(N_{X_{\mathbf{a}} / \mathbb{P}^{n-1}}\right)=\prod_{r=1}^{l}\left(1+\left.a_{r} \mathrm{H}\right|_{X_{\mathbf{a}}}\right) .
$$

It remains to verify the third identity. For each $r \in \mathbb{Z}^{\geq 0}$, let

$$
Z_{r}(Q) \equiv \sum_{d=1}^{\infty} Q^{d}\left\langle\psi_{1}^{r} \operatorname{ev}_{1}^{*} \mathrm{H}^{n-3-r} e\left(\mathcal{V}_{(1)}^{\prime}\right),\left[\overline{\mathfrak{M}}_{(1)}\left(\mathbb{P}^{n-1}, d\right)\right]\right\rangle .
$$

By (2.1), (2.2), and the decomposition along the small diagonal in $\left(\mathbb{P}^{n-1}\right)^{m}$, the left-hand side of the third identity in Lemma 2.1 above equals

$$
\begin{aligned}
\langle\mathbf{a}\rangle \sum_{d=1}^{\infty} & Q^{d}\left(\sum_{m=1}^{2 m \leq p} \frac{(-1)^{m}}{m}\left\langle\mathrm{ev}_{1}^{*} \mathrm{H}^{n-1-p} \prod_{i=1}^{m} \pi_{i}^{*} \frac{e\left(\mathcal{V}_{(1)}^{\prime}\right)}{1-\psi_{1}},\left[\overline{\mathfrak{M}}_{(m)}\left(\mathbb{P}^{n-1}, d\right)\right]\right\rangle\right) \\
= & \langle\mathbf{a}\rangle \sum_{m=1}^{2 m \leq p} \frac{(-1)^{m}}{m} \sum_{d=1}^{\infty} Q^{d} \\
& \times \sum_{\substack{i=1 \\
\sum_{i=1} d_{i}=d}} \sum_{\substack{i=1 \\
d_{i}>0}} \prod_{i=1}^{m}\left\langle\mathrm{ev}_{1}^{*} \mathrm{H}^{n-1-p_{i}} \frac{e\left(\mathcal{V}_{(1)}^{\prime}\right)}{1-\psi_{1}},\left[\overline{\mathfrak{M}}_{(1)}\left(\mathbb{P}^{n-1}, d_{i}\right)\right]\right\rangle \\
= & \langle\mathbf{a}\rangle \sum_{m=1}^{2 m \leq p} \frac{(-1)^{m}}{m} \\
& \times \sum_{\substack{m=1 \\
\sum_{i=1}^{m} p_{i}=p}} \prod_{i=1}^{m} Z_{p_{i}-2}(Q)=-\langle\mathbf{a}\rangle \llbracket \log \left(1+\sum_{r=0}^{n-3} Z_{r}(Q) w^{r+2}\right) \rrbracket_{w ; p} .
\end{aligned}
$$

The third statement of Lemma 2.1 now follows from

$$
1+\sum_{r=0}^{n-3} Z_{r}(Q) w^{r+2}=\mathrm{e}^{-J(q) w} \frac{\tilde{\mathcal{F}}(w, q)}{I_{0}(q)} \in \mathbb{Q}[w][[q]] / w^{n} ;
$$

the last identity is obtained from [Gi, Theorem 11.8] using the string relation MirSym, Section 26.3].

\section{EqUiVARIANT SETUP}

3.1. Equivariant cohomology. This section reviews the basics of equivariant cohomology following [Z5, Section 1.1] closely and setting up related notation.

The quotient of the classifying space for the $n$-torus $\mathbb{T}$ is $B \mathbb{T} \equiv\left(\mathbb{P}^{\infty}\right)^{n}$. Thus, the group cohomology of $\mathbb{T}$ is

$$
H_{\mathbb{T}}^{*} \equiv H^{*}(B \mathbb{T})=\mathbb{Q}\left[\alpha_{1}, \ldots, \alpha_{n}\right],
$$

where $\alpha_{i} \equiv \pi_{i}^{*} c_{1}\left(\gamma^{*}\right), \gamma \longrightarrow \mathbb{P}^{\infty}$ is the tautological line bundle, and $\pi_{i}:\left(\mathbb{P}^{\infty}\right)^{n} \longrightarrow \mathbb{P}^{\infty}$ is the projection to the $i$-th component. In the remainder of the paper,

$$
\alpha=\left(\alpha_{1}, \ldots, \alpha_{n}\right) .
$$


The field of fractions of $H_{\mathbb{T}}^{*}$ will be denoted by

$$
\mathbb{Q}_{\alpha} \equiv \mathbb{Q}\left(\alpha_{1}, \ldots, \alpha_{n}\right) \text {. }
$$

We denote the equivariant $\mathbb{Q}$-cohomology of a topological space $M$ with a $\mathbb{T}$-action by $H_{\mathbb{T}}^{*}(M)$. If the $\mathbb{T}$-action on $M$ lifts to an action on a complex vector bundle $V \longrightarrow M$, let $\mathbf{e}(V) \in H_{\mathbb{T}}^{*}(M)$ denote the equivariant Euler class of $V$. A continuous T-equivariant map $f: M \longrightarrow M^{\prime}$ between two compact oriented manifolds induces a pushforward homomorphism

$$
f_{*}: H_{\mathbb{T}}^{*}(M) \longrightarrow H_{\mathbb{T}}^{*}\left(M^{\prime}\right)
$$

which is characterized by the property that

$$
\int_{M^{\prime}}\left(f_{*} \eta\right) \eta^{\prime}=\int_{M} \eta\left(f^{*} \eta^{\prime}\right) \forall \eta \in H_{\mathbb{T}}^{*}(M), \eta^{\prime} \in H_{\mathbb{T}}^{*}\left(M^{\prime}\right) .
$$

If $M^{\prime}$ is a point, this is the integration-along-the-fiber homomorphism

$$
\int_{M}: H_{\mathbb{T}}^{*}(M) \longrightarrow H_{\mathbb{T}}^{*}
$$

for the fiber bundle $E \mathbb{T} \times_{\mathbb{T}} M \longrightarrow B \mathbb{T}$.

Throughout this paper, $\mathbb{T}$ will act on $\mathbb{P}^{n-1}$ in the standard way:

$$
\left(\mathrm{e}^{\mathrm{i} \theta_{1}}, \ldots, \mathrm{e}^{\mathrm{i} \theta_{n}}\right) \cdot\left[z_{1}, \ldots, z_{n}\right]=\left[\mathrm{e}^{\mathrm{i} \theta_{1}} z_{1}, \ldots, \mathrm{e}^{\mathrm{i} \theta_{n}} z_{n}\right] .
$$

This action has $n$ fixed points:

$$
P_{1}=[1,0, \ldots, 0], \quad P_{2}=[0,1,0, \ldots, 0], \quad \ldots, \quad P_{n}=[0, \ldots, 0,1] .
$$

For each $i=1,2, \ldots, n$, let

$$
\phi_{i} \equiv \prod_{k \neq i}\left(x-\alpha_{k}\right) \in H_{\mathbb{T}}^{*}\left(\mathbb{P}^{n-1}\right) .
$$

By the Atiyah-Bott localization theorem [ABo,

$$
\left.\eta\right|_{P_{i}}=\int_{\mathbb{P}^{n-1}} \eta \phi_{i} \in \mathbb{Q}_{\alpha} \quad \forall \eta \in H_{\mathbb{T}}^{*}\left(\mathbb{P}^{n-1}\right) \otimes_{H_{\mathbb{T}}^{*}} \mathbb{Q}_{\alpha}, i=1,2, \ldots, n ;
$$

thus, $\phi_{i}$ is the equivariant Poincaré dual of $P_{i}$.

The standard action of $\mathbb{T}$ on $\mathbb{P}^{n-1}$ lifts to an action on the tautological bundle

$$
\gamma \equiv \mathcal{O}_{\mathbb{P}^{n-1}}(-1) \subset \mathbb{P}^{n-1} \times \mathbb{C}^{n}
$$

by restricting the standard diagonal $\mathbb{T}$-action on $\mathbb{P}^{n-1} \times \mathbb{C}^{n}$. The equivariant hyperplane class is defined to be

$$
x \equiv \mathbf{e}\left(\gamma^{*}\right) \equiv \mathbf{e}\left(\mathcal{O}_{\mathbb{P}^{n-1}}(1)\right) \in H_{\mathbb{T}}^{2}\left(\mathbb{P}^{n-1}\right) .
$$

The equivariant cohomology of $\mathbb{P}^{n-1}$ is given by

$$
H_{\mathbb{T}}^{*}\left(\mathbb{P}^{n-1}\right)=\mathbb{Q}\left[x, \alpha_{1}, \ldots, \alpha_{n}\right] /\left(x-\alpha_{1}\right) \ldots\left(x-\alpha_{n}\right) .
$$

The restriction map on the equivariant cohomology induced by the inclusion $P_{i} \longrightarrow \mathbb{P}^{n-1}$ is given by

$$
H_{\mathbb{T}}^{*}\left(\mathbb{P}^{n-1}\right)=\mathbb{Q}\left[x, \alpha_{1}, \ldots, \alpha_{n}\right] / \prod_{k=1}^{n}\left(x-\alpha_{k}\right) \longrightarrow H_{\mathbb{T}}^{*}\left(P_{i}\right)=\mathbb{Q}\left[\alpha_{1}, \ldots, \alpha_{n}\right], \quad x \longrightarrow \alpha_{i},
$$

and so

$$
\eta=\left.0 \in H_{\mathbb{T}}^{*}\left(\mathbb{P}^{n-1}\right) \quad \Longleftrightarrow \quad \eta\right|_{P_{i}}=0 \in H_{\mathbb{T}}^{*} \forall i=1,2, \ldots, n .
$$


3.2. Generating function for reduced genus 1 GW invariants. As in [Z5, the reduced genus $1 \mathrm{GW}$ invariants $N_{1}^{d ; 0}\left(X_{\mathbf{a}}\right)$ of $X_{\mathbf{a}}$ are packaged into a generating function $\mathfrak{X}$; this is a power series in the formal variable $Q$ with coefficients in the equivariant cohomology of $\mathbb{P}^{n-1}$. In this section, we define $\mathfrak{X}$ and explain what its relationship with $N_{1}^{d ; 0}\left(X_{\mathbf{a}}\right)$ is; see (3.8) and (3.9).

Let $\pi: \mathfrak{U} \longrightarrow \overline{\mathfrak{M}}_{g, k}\left(\mathbb{P}^{n-1}, d\right)$ be the universal curve with evaluation map ev as before, and let

$$
\mathcal{V}_{0} \longrightarrow \overline{\mathfrak{M}}_{0, k}\left(\mathbb{P}^{n-1}, d\right)
$$

be the vector bundle corresponding to the locally free sheaf

$$
\bigoplus_{r=1}^{l} \pi_{*} \operatorname{ev}^{*} \mathcal{O}_{\mathbb{P}^{n-1}}\left(a_{r}\right) \longrightarrow \overline{\mathfrak{M}}_{0, k}\left(\mathbb{P}^{n-1}, d\right) .
$$

The Euler class $e\left(\mathcal{V}_{0}\right)$ relates genus $0 \mathrm{GW}$ invariants of $X_{\mathbf{a}} \subset \mathbb{P}^{n-1}$ to genus $0 \mathrm{GW}$ invariants of $\mathbb{P}^{n-1}$; it also appears in the genus 0 two point generating functions (3.11) - (3.13), which are used in the proof in Theorem 3 .

The genus $1 \mathrm{GW}$ invariants of $X_{\mathbf{a}}$ are related to the $\mathrm{GW}$ invariants of $\mathbb{P}^{n-1}$ in a more complicated way. This is partly because $\overline{\mathfrak{M}}_{1, k}\left(\mathbb{P}^{n-1}, d\right)$ is not an orbifold and

$$
\bigoplus_{r=1}^{l} \pi_{*} \operatorname{ev}^{*} \mathcal{O}_{\mathbb{P}^{n-1}}\left(a_{r}\right) \longrightarrow \overline{\mathfrak{M}}_{1, k}\left(\mathbb{P}^{n-1}, d\right)
$$

is not locally free. However, it is shown in $\mathrm{VaZ}$ that there exists a natural desingularization

$$
p: \widetilde{\mathfrak{M}}_{1, k}^{0}\left(\mathbb{P}^{n-1}, d\right) \longrightarrow \overline{\mathfrak{M}}_{1, k}^{0}\left(\mathbb{P}^{n-1}, d\right)
$$

of the main component of $\overline{\mathfrak{M}}_{1, k}\left(\mathbb{P}^{n-1}, d\right)$, whose generic element is a map from a smooth domain. There is also a vector orbi-bundle $\mathcal{V}_{1}$ over $\widetilde{\mathfrak{M}}_{1, k}^{0}\left(\mathbb{P}^{n-1}, d\right)$ so that the diagram

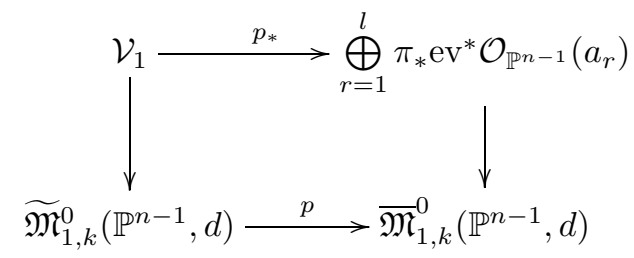

commutes. By [LiZ, Theorem 1.1] and [Z2, Theorem 1.1],

$$
d N_{1}^{d ; 0}\left(X_{\mathbf{a}}\right)=\left\langle e\left(\mathcal{V}_{1}\right) \mathrm{ev}_{1}^{*} \mathrm{H},\left[\widetilde{\mathfrak{M}}_{1,1}^{0}\left(\mathbb{P}^{n-1}, d\right)\right]\right\rangle .
$$

The standard $\mathbb{T}$-action on $\mathbb{P}^{n-1}$ induces $\mathbb{T}$-actions on the moduli spaces $\overline{\mathfrak{M}}_{g, k}\left(\mathbb{P}^{n-1}, d\right)$ and lifts to an action on $\widetilde{\mathfrak{M}}_{1, k}^{0}\left(\mathbb{P}^{n-1}, d\right)$. The evaluation maps,

$\mathrm{ev}_{i}: \overline{\mathfrak{M}}_{g, k}\left(\mathbb{P}^{n-1}, d\right), \widetilde{\mathfrak{M}}_{1, k}^{0}\left(\mathbb{P}^{n-1}, d\right) \longrightarrow \mathbb{P}^{n-1}, \quad\left[\mathcal{C}, y_{1}, \ldots, y_{k}, u\right] \longrightarrow u\left(y_{i}\right), \quad i \in[k]$,

are $\mathbb{T}$-equivariant. The natural $\mathbb{T}$-action on $\mathcal{O}_{\mathbb{P}^{n-1}}(-1) \longrightarrow \mathbb{P}^{n-1}$ induces $\mathbb{T}$-actions on the sheafs $\pi_{*} \mathrm{ev}^{*} \mathcal{O}_{\mathbb{P}^{n-1}}(a)$ and the vector bundle

$$
\mathcal{V}_{1} \longrightarrow \widetilde{\mathfrak{M}}_{1,1}^{0}\left(\mathbb{P}^{n-1}, d\right)
$$


With ev $1, d$ the evaluation map on $\widetilde{\mathfrak{M}}_{1,1}^{0}\left(\mathbb{P}^{n-1}, d\right)$, let

$$
\mathfrak{X}(\alpha, x, Q) \equiv \sum_{d=1}^{\infty} Q^{d}\left(\operatorname{ev}_{1, d *} \mathbf{e}\left(\mathcal{V}_{1}\right)\right) \in\left(H_{\mathbb{T}}^{2(n-2)}\left(\mathbb{P}^{n-1}\right)\right)[[Q]] .
$$

By (3.4),

$$
\mathfrak{X}(\alpha, x, Q)=\mathfrak{X}_{0}(Q) x^{n-2}+\mathfrak{X}_{1}(\alpha, Q) x^{n-3}+\ldots+\mathfrak{X}_{n-2}(\alpha, Q) x^{0},
$$

for some $\mathfrak{X}_{0} \in \mathbb{Q}[[Q]]$ and power series $\mathfrak{X}_{p} \in \mathbb{Q}\left[\alpha_{1}, \ldots, \alpha_{n}\right][[Q]]$, whose coefficients are symmetric degree $p$ homogeneous polynomials in $\alpha_{1}, \ldots, \alpha_{n}$. By (3.7) and (3.1),

$$
Q \frac{\mathrm{d}}{\mathrm{d} Q} \sum_{d=1}^{\infty} N_{1}^{d ; 0}\left(X_{\mathbf{a}}\right) Q^{d}=\mathfrak{X}_{0}(Q) .
$$

By (3.5), (3.3), and (3.1),

$$
\begin{aligned}
\mathfrak{X}\left(\alpha, \alpha_{i}, Q\right) & =\left.\mathfrak{X}(\alpha, x, Q)\right|_{P_{i}}=\sum_{d=1}^{\infty} Q^{d} \int_{\mathbb{P}^{n-1}}\left(\mathrm{ev}_{1, d *} \mathbf{e}\left(\mathcal{V}_{1}\right)\right) \phi_{i} \\
& =\sum_{d=1}^{\infty} Q^{d} \int_{\widetilde{\mathfrak{M}}_{1,1}^{0}\left(\mathbb{P}^{n-1}, d\right)} \mathbf{e}\left(\mathcal{V}_{1}\right) \mathrm{ev}_{1}^{*} \phi_{i} \in \mathbb{Q}_{\alpha}[[Q]]
\end{aligned}
$$

for each $i=1,2, \ldots, n$. Since $\mathfrak{X}$ is symmetric in $\alpha_{1}, \ldots, \alpha_{n}, \mathfrak{X}_{0} \in \mathbb{Q}[[Q]]$ is completely determined by either of the $n$ power series in (3.10). We use this to obtain the explicit formula for $\mathfrak{X}_{0}$ given in Theorem 3 .

3.3. A localization proposition. As in [Z5], we express $\mathfrak{X}\left(\alpha, \alpha_{i}, Q\right)$ in terms of residues of genus 0 generating functions. Proposition 3.1 below is the analogue of [Z5. Propositions 1.1, 1.2]; its proof is essentially identical to the proof of [Z5, Propositions 1.1, 1.2] in [Z5, Section 2]. In this section, we set up the notation needed to state Proposition 3.1 motivate it, and describe the few minor changes needed in [Z5, Section 2] for a complete proof of this proposition. In the remainder of this paper, we will use Proposition 3.1 to obtain an explicit formula for $\mathfrak{X}_{0}$.

If $f$ is a rational function in $\hbar$ and possibly other variables and $\hbar_{0} \in S^{2}$, let $\Re_{\hbar=\hbar_{0}} f(\hbar)$ denote the residue of the one-form $f(\hbar) d \hbar$ at $\hbar=\hbar_{0}$; thus,

$$
\mathfrak{R}_{\hbar=\infty} f(\hbar) \equiv-\mathfrak{R}_{w=0}\left\{w^{-2} f\left(w^{-1}\right)\right\} .
$$

If $f$ involves variables other than $\hbar, \Re_{\hbar=\hbar_{0}} f(\hbar)$ is a function of the other variables. If $f$ is a power series in $Q$ with coefficients that are rational functions in $\hbar$ and possibly other variables, let $\Re_{\hbar=\hbar_{0}} f(\hbar)$ denote the power series in $Q$ obtained by replacing each of the coefficients by its residue at $\hbar=\hbar_{0}$. If $\hbar_{1}, \ldots, \hbar_{k}$ is a collection of points in $S^{2}$, not necessarily distinct, we define

$$
\mathfrak{R}_{\hbar=\hbar_{1}, \ldots, \hbar_{k}} f(\hbar) \equiv \sum_{z \in\left\{\hbar_{1}, \ldots, \hbar_{k}\right\}} \Re_{\hbar=z} f(\hbar) .
$$

If $\hbar_{0} \in \mathbb{C}$ or $\hbar_{0}$ is one of the "other" variables in $f$, let

$$
\mathfrak{R}_{\hbar=-\mathbf{a} \hbar_{0}} f(\hbar) \equiv \mathfrak{R}_{\hbar=-a_{1} \hbar_{0}, \ldots,-a_{l} \hbar_{0}} f(\hbar) .
$$

For instance, if $\mathbf{a}=(2,2,3,3,3,3)$ and $\alpha_{i}$ is one of the other variables, then

$$
\mathfrak{R}_{\hbar=-\mathbf{a} \alpha_{i}} f(\hbar) \equiv \mathfrak{R}_{\hbar=-2 \alpha_{i}} f(\hbar)+\mathfrak{R}_{\hbar=-3 \alpha_{i}} f(\hbar) .
$$


Since the $\mathbb{T}$-equivariant bundle homomorphism

$$
\widetilde{\mathrm{ev}}_{1}: \mathcal{V}_{0} \longrightarrow \bigoplus_{r=1}^{l} \mathrm{ev}_{1}^{*} \mathcal{O}_{\mathbb{P}^{n-1}}\left(a_{r}\right), \quad\left[\mathcal{C}, y_{1}(\mathcal{C}), \ldots, y_{k}(\mathcal{C}), u, \xi\right] \longrightarrow \xi\left(y_{1}(\mathcal{C})\right)
$$

is surjective, its kernel

$$
\mathcal{V}_{0}^{\prime} \equiv \operatorname{ker} \widetilde{\mathrm{ev}}_{1} \longrightarrow \overline{\mathfrak{M}}_{0, k}\left(\mathbb{P}^{n-1}, d\right),
$$

is a $\mathbb{T}$-equivariant vector bundle. Since the $\mathbb{T}$-action on $\overline{\mathfrak{M}}_{g, k}\left(\mathbb{P}^{n-1}, d\right)$ lifts naturally to the tautological tangent line bundles $L_{i}$, there are well-defined equivariant $\psi$ classes

$$
\psi_{i} \equiv c_{1}\left(L_{i}^{*}\right) \in H_{\mathbb{T}}^{*}\left(\overline{\mathfrak{M}}_{g, k}\left(\mathbb{P}^{n-1}, d\right)\right) ;
$$

see MirSym, Section 25.2]. For all $i, j=1,2, \ldots, n$, let

$$
\begin{aligned}
\mathcal{Z}_{i}^{*}(\hbar, Q) & \equiv \sum_{d=1}^{\infty} Q^{d} \int_{\overline{\mathfrak{M}}_{0,2}\left(\mathbb{P}^{n-1}, d\right)} \frac{\mathbf{e}\left(\mathcal{V}_{0}^{\prime}\right)}{\hbar-\psi_{1}} \mathrm{ev}_{1}^{*} \phi_{i}, \\
\mathcal{Z}_{i j}^{*}(\hbar, Q) & \equiv \hbar^{-1} \sum_{d=1}^{\infty} Q^{d} \int_{\overline{\mathfrak{M}}_{0,2}\left(\mathbb{P}^{n-1}, d\right)} \frac{\mathbf{e}\left(\mathcal{V}_{0}^{\prime}\right)}{\hbar-\psi_{1}} \mathrm{ev}_{1}^{*} \phi_{i} \mathrm{ev}_{2}^{*} \phi_{j}, \\
\widetilde{\mathcal{Z}}_{i j}^{*}\left(\hbar_{1}, \hbar_{2}, Q\right) & \equiv \frac{1}{2 \hbar_{1} \hbar_{2}} \sum_{d=1}^{\infty} Q^{d} \int_{\overline{\mathfrak{M}}_{0,2}\left(\mathbb{P}^{n-1}, d\right)} \frac{\mathbf{e}\left(\mathcal{V}_{0}^{\prime}\right)}{\left(\hbar_{1}-\psi_{1}\right)\left(\hbar_{2}-\psi_{2}\right)} \operatorname{ev}_{1}^{*} \phi_{i} \mathrm{ev}_{2}^{*} \phi_{j} .
\end{aligned}
$$

Explicit formulas for these generating functions are given in [Gi, Theorem 11.8] and [PoZ, Theorem 6]. These theorems show that in particular

$$
\mathcal{Z}_{i}^{*}, \mathcal{Z}_{i j}^{*} \in \mathbb{Q}_{\alpha}(\hbar)[[Q]] \quad \text { and } \quad \widetilde{\mathcal{Z}}_{i j}^{*} \in \mathbb{Q}_{\alpha}\left(\hbar_{1}, \hbar_{2}\right)[[Q]] .
$$

Thus, the $\hbar$-residues of these power series are well defined. Since the $Q$ degree 0 term of the power series $\mathcal{Z}_{i}^{*}(\hbar, Q)$ is 0 , the residue

$$
\eta_{i}(Q) \equiv \Re_{\hbar=0}\left\{\log \left(1+\mathcal{Z}_{i}^{*}(\hbar, Q)\right)\right\} \in \mathbb{Q}_{\alpha}[[Q]]
$$

is well defined. Let

$$
\Phi_{0}\left(\alpha_{i}, Q\right) \equiv \mathfrak{R}_{\hbar=0}\left\{\hbar^{-1} \mathrm{e}^{-\eta_{i}(Q) / \hbar}\left(1+\mathcal{Z}_{i}^{*}(\hbar, Q)\right)\right\} \in \mathbb{Q}_{\alpha}[[Q]] .
$$

By [Z5, Lemma 2.3], the power series $\mathrm{e}^{-\eta_{i}(Q) / \hbar}\left(1+\mathcal{Z}_{i}^{*}(\hbar, Q)\right)$ is holomorphic at $\hbar=0$; thus $\Phi_{0}\left(\alpha_{i}, Q\right)$ is its value at $\hbar=06$ Note that the degree 0 term of $\Phi_{0}\left(\alpha_{i}, Q\right)$ is 1 .

Proposition 3.1 below is obtained by applying the Atiyah-Bott localization theorem [ABO to the last expression in (3.10). As described in detail in $\mathrm{Z5}$. Sections $1.3,1.4]$, the fixed loci of the $\mathbb{T}$-action on $\widetilde{\mathfrak{M}}_{1,1}^{0}\left(\mathbb{P}^{n-1}, d\right)$ are indexed by decorated graphs with one marked point. The vertices are decorated by elements of $[n]$, indicating the $\mathbb{T}$-fixed point of $\mathbb{P}^{n-1}$ to which the node, ramification point, or component corresponding to the vertex is mapped. These graphs have either zero loops and one distinguished vertex (as in Figure 2) or one loop (as in Figure 1), depending on whether the stable maps they describe are constant or not on the principal component of the domain 7 The graphs with no loops are called $B$-graphs in [Z5], while the graphs with one loop are called $A$-graphs. In a $B$-graph, the distinguished

\footnotetext{
${ }^{6}$ While $l$ is meant to be 1 in [Z5, Section 2.2], the argument goes through for any a without any change.

${ }^{7}$ Figures 1 and 2 are Figures 1 and 4 in $[\mathrm{Z5}$; they are used by permission to indicate what is involved in the proof of (the $l=1$ case of) Proposition 3.1 in [Z5].
} 


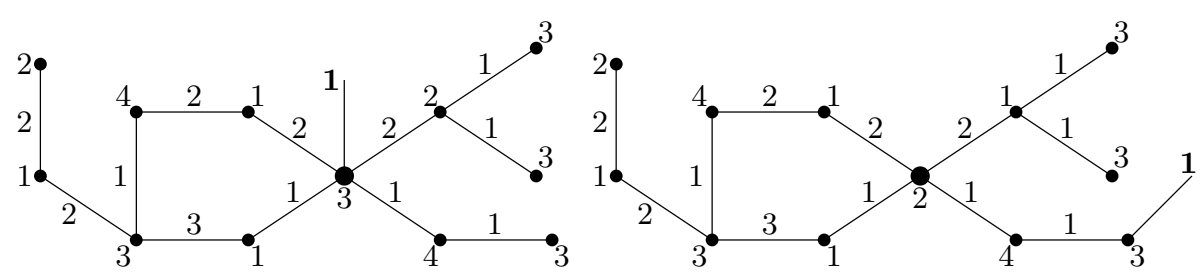

FiguRE 1. Decorated graphs of types $A_{3}$ and $\tilde{A}_{32}$

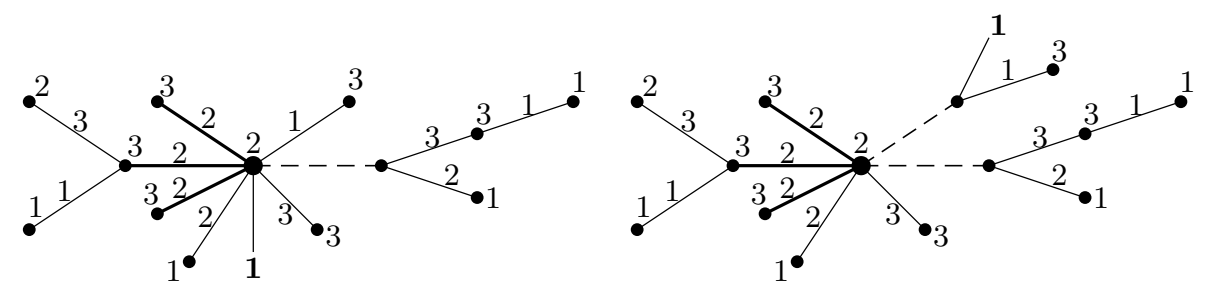

Figure 2. Decorated rooted trees of types $B_{2}$ and $\tilde{B}_{22}$

vertex corresponds to the contracted principal component. As every graph has a marked point, even the $A$-graphs have a distinguished vertex: the vertex $i n$ the loop closest to the vertex to which the marked point is attached. The distinguished vertices are indicated by thick dots in the four graphs in Figures 1 1 and 2 ,

Within each of the two types, there are two subtypes of graphs, depending on whether the marked point is attached to the distinguished vertex or some other vertex. In the former case, a graph has one special vertex label: the number decorating the vertex to which the marked point is attached. In the latter case, a graph has two special vertex labels: the number decorating the vertex to which the marked point is attached and the number decorating the distinguished vertex. Since $\left.\phi_{i}\right|_{P_{j}}=\delta_{i j}$, only the graphs that describe stable maps taking the marked point to $P_{i}$ contribute to (3.10); in these graphs the first special vertex label is $i$. Thus, the types of graphs that contribute to (3.10) can be described as $A_{i}, \tilde{A}_{i j}$, $B_{i}$, and $\tilde{B}_{i j}$, with the first subscript describing the label of the vertex to which the marked point is attached and the second describing the label of the distinguished vertex if this vertex is different from the first (the label may still be the same).

The approach of [Z5] to computing the total contribution to (3.10) of all graphs of a fixed type is to break every graph at the distinguished vertex, adding a marked point to each of the resulting "strands" so that each graph is completely encoded by its strands. In the case of $B_{i}$-graphs, all strands are graphs with one marked point. In the case of $A_{i^{-}}$and $B_{i j}$-graphs, there is precisely one strand with two marked points; in the former case it contributes to $\widetilde{\mathcal{Z}}_{i i}^{*}$, while in the latter it contributes to $\mathcal{Z}_{j i}^{*}$. In the case of $A_{i j}$-graphs, there are two strands with two marked points, one of which contributes to $\widetilde{\mathcal{Z}}_{j j}^{*}$, while the other to $\mathcal{Z}_{j i}^{*}$. Each of the one-pointed strands contributes to $\mathcal{Z}_{j}^{*}$. While the number of one-pointed strands can be arbitrarily large, it is possible to sum up over all arrangements of such strands because of a special 
property of the power series $\mathcal{Z}_{i}^{*}$ described in [Z5, Section 2.2]. This reduces the total contribution, $\mathcal{A}_{i}, \tilde{\mathcal{A}}_{i j}, \mathcal{B}_{i}$, or $\tilde{\mathcal{B}}_{i j}$ of all graphs of a fixed type, $A_{i}, \tilde{A}_{i j}, B_{i}$, or $\tilde{B}_{i j}$, to a fairly simple expression involving $\mathcal{Z}_{i}^{*}, \mathcal{Z}_{i j}^{*}$, and/or $\widetilde{\mathcal{Z}}_{i j}^{*}$.

Proposition 3.1. For every $i=1,2, \ldots, n$,

$$
\mathfrak{X}\left(\alpha, \alpha_{i}, Q\right)=\mathcal{A}_{i}(Q)+\sum_{j=1}^{n} \tilde{\mathcal{A}}_{i j}(Q)+\mathcal{B}_{i}(Q)+\sum_{j=1}^{n} \tilde{\mathcal{B}}_{i j}(Q),
$$

where

$$
\begin{aligned}
& \mathcal{A}_{i}(Q)=\frac{1}{\Phi_{0}\left(\alpha_{i}, Q\right)} \Re_{\hbar_{1}=0}\left\{\mathfrak{R}_{\hbar_{2}=0}\left\{\mathrm{e}^{-\eta_{i}(Q) / \hbar_{1}} \mathrm{e}^{-\eta_{i}(Q) / \hbar_{2}} \widetilde{\mathcal{Z}}_{i i}^{*}\left(\hbar_{1}, \hbar_{2}, Q\right)\right\}\right\} \\
& \tilde{\mathcal{A}}_{i j}(Q)=\frac{\mathcal{A}_{j}(Q)}{\prod_{k \neq j}\left(\alpha_{j}-\alpha_{k}\right)} \Re_{\hbar=0}\left\{e^{-\eta_{j}(Q) / \hbar} \mathcal{Z}_{j i}^{*}(\hbar, Q)\right\} \\
& \mathcal{B}_{i}(Q)=\frac{\langle\mathbf{a}\rangle \alpha_{i}^{l}}{24} \Re_{\hbar=0, \infty,-\mathbf{a} \alpha_{i}}\left\{\frac{\prod_{k=1}^{n}\left(\alpha_{i}-\alpha_{k}+\hbar\right)}{\hbar^{3} \prod_{r=1}^{l}\left(a_{r} \alpha_{i}+\hbar\right)} \frac{\mathcal{Z}_{i}^{*}(\hbar, Q)}{1+\mathcal{Z}_{i}^{*}(\hbar, Q)}\right\} ; \\
& \tilde{\mathcal{B}}_{i j}(Q)=-\frac{1}{\prod_{k \neq j}\left(\alpha_{j}-\alpha_{k}\right)} \frac{\langle\mathbf{a}\rangle \alpha_{j}^{l}}{24} \Re_{\hbar=0, \infty,-\mathbf{a} \alpha_{j}}\left\{\frac{\prod_{k=1}^{n}\left(\alpha_{j}-\alpha_{k}+\hbar\right)}{\hbar^{2} \prod_{r=1}^{l}\left(a_{r} \alpha_{j}+\hbar\right)} \frac{\mathcal{Z}_{j i}^{*}(\hbar, Q)}{1+\mathcal{Z}_{j}^{*}(\hbar, Q)}\right\}
\end{aligned}
$$

This proposition is essentially proved in $\mathrm{Z} 5$, Sections $1.3,1.4,2]$, which treats the $l=1$ case. In the general case, the $\mathbb{T}$-fixed loci and their normal bundles remain the same. The only required changes involve the Euler classes of the bundles $\mathcal{V}_{0}^{\prime}$ and $\mathcal{V}_{1}$, which are now products of the Euler classes of the bundles in [Z5]. These changes are as follows:

(i) Equation [Z5, (1.37)] becomes

$$
\left.\mathbf{e}\left(\mathcal{V}_{1}\right)\right|_{\widetilde{\mathcal{Z}}_{\Gamma}}=\langle\mathbf{a}\rangle \alpha_{\mu\left(v_{0}\right)}^{l} \prod_{e \in \operatorname{Edg}\left(v_{0}\right)} \pi_{e}^{*} \mathbf{e}\left(\mathcal{V}_{0}^{\prime}\right) / \prod_{r=1}^{l}\left(a_{r} \alpha_{\mu\left(v_{0}\right)}+\psi_{\Gamma}+\lambda\right) ;
$$

(ii) $n \alpha_{i}+\hbar$ is replaced by $\prod_{r=1}^{l}\left(a_{r} \alpha_{i}+\hbar\right)$ in the definition of $\Psi$ above [Z5, (2.19)], in [Z5, (2.23) and (2.24)], and in the last equation in [Z5, Section 2.3], leading to the corresponding modification in the final expressions for $\mathcal{B}_{i}$ and $\tilde{\mathcal{B}}_{i j}$ above;

(iii) $n \alpha_{i}$ is replaced by $\langle\mathbf{a}\rangle \alpha_{i}^{l}$ in $[\mathrm{Z5},(2.24)]$ and $n \alpha_{j}$ is replaced by $\langle\mathbf{a}\rangle \alpha_{j}^{l}$ in the last equation in [Z5, Section 2.3], leading to the corresponding modification in the final expressions for $\mathcal{B}_{i}$ and $\tilde{\mathcal{B}}_{i j}$ above.

\section{SOME PROPERTIES OF HYPERGEOMETRIC SERIES $\tilde{\mathcal{F}}$}

In this section we study properties of the hypergeometric series $\tilde{\mathcal{F}}$ of (1.2) which are used in Section 5.3 to deduce Theorem 3 from Proposition 3.1. The results in this section extend most of the statements and proofs in [ZaZ], which treats the $l=1$ case. 
Let $\mathbf{M}: \mathcal{P} \longrightarrow \mathcal{P}$ be as in (1.3) and define $\mathcal{F} \in \mathcal{P}$ by

$$
\mathcal{F}(w, q) \equiv \sum_{d=0}^{\infty} q^{d} \frac{\prod_{k=1}^{l} \prod_{r=1}^{a_{k} d}\left(a_{k} w+r\right)}{\prod_{r=1}^{d}\left[(w+r)^{n}-w^{n}\right]} .
$$

By (1.2) and (1.4)

$$
I_{p}(q) \equiv \mathbf{M}^{p} \tilde{\mathcal{F}}(0, q)=\mathbf{M}^{p} \mathcal{F}(0, q) \quad \forall p=0,1, \ldots, n-1 .
$$

Some advantages of the power series $\mathcal{F}$ over $\tilde{\mathcal{F}}$ are illustrated by Lemmas 4.1 and 4.2 below.

Lemma 4.1. The hypergeometric series $\mathcal{F}$ satisfies $\mathbf{M}^{n} \mathcal{F}=\mathcal{F}$.

Lemma 4.2 ([ZaZ, Lemma 1.3 and its proof $]$ ). If $F \in \mathcal{P}$ and $\mathbf{M}^{k} F=F$ for some $k>0$, then every coefficient of the power series $\log \mathbf{M}^{p} F(w, q) \in \mathbb{Q}(w)[[q]]$ is $\mathcal{O}(w)$ as $w \rightarrow \infty$ for all $p \geq 0$. Moreover, $\mathfrak{R}_{w=0}\left\{\log \mathbf{M}^{p} F\left(w^{-1}, q\right)\right\}$ does not depend on $p$.

Applying this lemma to $F=\mathcal{F}$, we find that $\mathcal{F}(w, q)$ has an asymptotic expansion

$$
\mathcal{F}(w, q) \sim \mathrm{e}^{\mu(q) w} \sum_{s=0}^{\infty} \Phi_{s}(q) w^{-s} \quad(w \rightarrow \infty)
$$

for some power series $\mu, \Phi_{0}, \Phi_{1}, \ldots$ in $\mathbb{Q}[[q]]$. Let

$$
L(q) \equiv\left(1-\mathbf{a}^{\mathbf{a}} q\right)^{-1 / n} \in \mathbb{Q}[[q]] .
$$

Proposition 4.3. The power series $\mu, \Phi_{0}$, and $\Phi_{1}$ in (4.3) are given by

$$
\begin{gathered}
\mu(q)=\int_{0}^{q} \frac{L(u)-1}{u} \mathrm{~d} u, \quad \Phi_{0}=L^{\frac{l+1}{2}}, \\
\Phi_{1}=\left\{\left[\frac{n-1}{24}-\frac{1}{12} \sum_{r=1}^{l} \frac{1}{a_{r}}\right](L-1)-\frac{(n-2)(n+1)-3\left(l^{2}-1\right)}{24 n}\left(L^{n}-1\right)\right\} \frac{\Phi_{0}}{L} .
\end{gathered}
$$

The last proposition of this section concerns properties of $\tilde{\mathcal{F}}$ and $\mathcal{F}$ around $w=0$.

Proposition 4.4. The power series $I_{p}(q)$ defined by (1.4) satisfy

$$
\begin{aligned}
& I_{p}(q)=1 \quad \forall p=n-l+1, \ldots, n-1, \\
& I_{p}(q)=I_{n-l-p}(q) \quad \forall p=0,1, \ldots, n-l, \\
& I_{0}(q) I_{1}(q) \ldots I_{n-l}(q)=\left(1-\mathbf{a}^{\mathbf{a}} q\right)^{-1}, \\
& I_{0}(q)^{n-l} I_{1}(q)^{n-l-1} \ldots I_{n-l-1}(q)^{1} I_{n-l}(q)^{0}=\left(1-\mathbf{a}^{\mathbf{a}} q\right)^{-(n-l) / 2} .
\end{aligned}
$$

While (4.8) and (4.9) imply (4.10), (4.10) is simpler to prove directly than (4.8) and will be verified together with (4.9), as is done in [ZaZ].

Lemma 4.1 and Propositions 4.3 and 4.4 are proved in Sections 4.14 .3 following the approach in $\mathrm{ZaZ}$. Let

$$
D=q \frac{\mathrm{d}}{\mathrm{d} q}, D_{w}=D+w: \mathbb{Q}(w)[[q]] \longrightarrow \mathbb{Q}(w)[[q]] .
$$


Thus,

$$
\begin{aligned}
D_{w}\left[\sum_{d=0}^{\infty} c_{d}(w) q^{d}\right] & =\sum_{d=0}^{\infty}(w+d) c_{d}(w) q^{d}, \\
\mathbf{M} F(w, q) & =w^{-1} D_{w}[F(w, q) / F(0, q)] \quad \forall F \in \mathcal{P} .
\end{aligned}
$$

4.1. Proof of Lemma 4.1 and Proposition 4.4. We will repeatedly use the following lemma.

Lemma 4.5 ([ZaZ, Corollary 2.2]). Suppose $F(w, q) \in \mathcal{P}$ satisfies

$$
\left(\sum_{r=0}^{m} C_{r}(q) D_{w}^{r}\right) F(w, q)=A(w, q)
$$

for some power series $C_{0}(q), \ldots, C_{m}(q) \in \mathbb{Q}[[q]]$ and $A(w, q) \in \mathbb{Q}(w)[[q]]$ with $A(0, q) \equiv 0$. Then

$$
\left(\sum_{s=0}^{m-1} \tilde{C}_{s}(q) D_{w}^{s}\right) \mathbf{M} F(w, q)=\frac{1}{w} A(w, q),
$$

where $\tilde{C}_{s}(q) \equiv \sum_{r=s+1}^{m}\left(\begin{array}{c}r \\ s+1\end{array}\right) C_{r}(q) D^{r-1-s} F(0, q)$.

Define power series $\mathcal{F}_{-l}, \mathcal{F}_{-l+1}, \ldots \in \mathcal{P}$ by

$$
\begin{aligned}
\mathcal{F}_{-l}(w, q) & \equiv \sum_{d=0}^{\infty} q^{d} \frac{\prod_{k=1}^{l} \prod_{r=0}^{a_{k} d-1}\left(a_{k} w+r\right)}{\prod_{r=1}^{d}\left[(w+r)^{n}-w^{n}\right]} \\
\mathcal{F}_{p} & \equiv \mathbf{M}^{l+p} \mathcal{F}_{-l} \quad \forall p>-l .
\end{aligned}
$$

Using (4.11), we find that

$$
\mathcal{F}_{p}(0, q)=1 \forall p=-l,-l+1, \ldots,-1, \quad w^{-1} D_{w} \mathcal{F}_{-1}=\mathcal{F} ;
$$

thus, $\mathcal{F}_{p}=\mathbf{M}^{p} \mathcal{F}$ for all $p \geq 0$.

It is also straightforward to check that $\mathcal{F}_{-l}$ solves the differential equation

$$
\left\{D_{w}^{n}-q \prod_{k=1}^{l} \prod_{r=0}^{a_{k}-1}\left(a_{k} D_{w}+r\right)\right\} \mathcal{F}_{-l}=w^{n} \mathcal{F}_{-l} .
$$

This equation is of the form (4.12) with $F=\mathcal{F}_{-l}, A=w^{n} \mathcal{F}_{-l}, m=n$,

$$
C_{n}(q)=1-\mathbf{a}^{\mathbf{a}} q, \quad C_{n-1}(q)=-\frac{n-l}{2} \mathbf{a}^{\mathbf{a}} q .
$$

Applying Lemma 4.5 repeatedly, we obtain

$$
\sum_{s=0}^{n-l-p} C_{s}^{(p)}(q) D_{w}^{s} \mathcal{F}_{p}(w, q)=w^{n-l-p} \mathcal{F}_{-l}(w, q), \quad-l \leq p \leq n-l,
$$


where by the first identity in (4.15) and by (4.2)

$$
\begin{aligned}
& C_{s}^{(p)}(q)=C_{s+l+p}^{(-l)}(q) \quad-l \leq p \leq 0, \\
& C_{s}^{(p)}(q)=\sum_{r=s+1}^{n-l-p+1}\left(\begin{array}{c}
r \\
s+1
\end{array}\right) C_{r}^{(p-1)}(q) D^{r-s-1} I_{p-1}(q) \quad p>0 .
\end{aligned}
$$

Using (4.17) and induction on $p$, we find that the top two coefficients in (4.18) are given by

$$
\begin{aligned}
C_{n-l-p}^{(p)} & =\left(1-\mathbf{a}^{\mathbf{a}} q\right) \prod_{r=0}^{p-1} I_{r}(q), \\
C_{n-l-p-1}^{(p)} & =\left[-\mathbf{a}^{\mathbf{a}} q \frac{n-l}{2}+\left(1-\mathbf{a}^{\mathbf{a}} q\right) \sum_{r=0}^{p-1}(n-l-r) \frac{D I_{r}(q)}{I_{r}(q)}\right] \prod_{r=0}^{p-1} I_{r}(q) .
\end{aligned}
$$

Setting $p=n-l$ in (4.18) and (4.19) thus gives

$$
\left(1-\mathbf{a}^{\mathbf{a}} q\right)\left(\prod_{r=0}^{n-1-l} I_{r}(q)\right) \mathcal{F}_{n-l}(w, q)=\mathcal{F}_{-l}(w, q) .
$$

Setting $w=0$ in (4.21) and using $\mathcal{F}_{-l}(0, q)=1$ gives (4.9). Substituting (4.9) back into (4.21) gives $\mathcal{F}_{n-l} / I_{n-l}=\mathcal{F}_{-l}$ and thus $\mathcal{F}_{n-l+1}=\mathcal{F}_{-l+1}$. Applying $\mathbf{M}$ to both sides of the last identity $l-1$ times and using (4.15), we obtain Lemma 4.1 and equation (4.7). Similarly, setting $p=n-l-1$ in (4.18)-(4.20) and then taking $w=0$ gives

$$
\sum_{r=0}^{n-1-l}(n-l-r) \frac{D I_{r}(q)}{I_{r}(q)}=\frac{n-l}{2} \frac{\mathbf{a}^{\mathbf{a}} q}{1-\mathbf{a}^{\mathbf{a}} q} .
$$

Integrating this identity and then exponentiating, we obtain (4.10).

We next prove the reflection symmetry (4.8). The function $\widetilde{\mathcal{F}} \in \mathcal{P}$ defined in (1.2) satisfies the differential equation

$$
\left\{D_{w}^{n-l}-\langle\mathbf{a}\rangle q \prod_{k=1}^{l} \prod_{r=1}^{a_{k}-1}\left(a_{k} D_{w}+r\right)\right\} \widetilde{\mathcal{F}}=w^{n-l} .
$$

This equation is of the form (4.12) with $F=\tilde{\mathcal{F}}, A=w^{n-l}, m=n-l$, and

$$
C_{n-l}(q)=1-\mathbf{a}^{\mathbf{a}} q .
$$

Applying Lemma 4.5 repeatedly, we obtain

$$
\sum_{s=0}^{n-l-p} \tilde{C}_{s}^{(p)}(q) D_{w}^{s} \mathbf{M}^{p} \widetilde{\mathcal{F}}(w, q)=w^{n-l-p}, \quad 0 \leq p \leq n-l,
$$

with $\tilde{C}_{n-l-p}^{(p)}=C_{n-l-p}^{(p)}$ given by (4.19). Setting $p=n-l$ in (4.22) and using (4.19) and (4.9), we find that

$$
\mathbf{M}^{n-l} \widetilde{\mathcal{F}}(w, q)=I_{n-l}(q)
$$


is independent of $w$. Using (4.11) and downward induction on $p$, we then find that

$$
w^{l-n} \widetilde{\mathcal{F}}(w, q)=I_{0} D_{w}^{-1} I_{1} D_{w}^{-1} \ldots I_{n-l-1} D_{w}^{-1} I_{n-l},
$$

where

$$
D_{w}^{-1}\left[\sum_{d=0}^{\infty} c_{d}(w) q^{d}\right]=\sum_{d=0}^{\infty} \frac{c_{d}(w)}{(w+d)} q^{d} .
$$

Comparing the coefficients of $q^{d}$ on the two sides of (4.23), we find that

$$
\begin{aligned}
& \frac{\langle\mathbf{a}\rangle^{-1} \prod_{k=1}^{l} \prod_{r=0}^{a_{k} d}\left(a_{k} w+r\right)}{[w(w+1) \ldots(w+d)]^{n}} \\
& \quad=\sum_{\substack{d_{0}+\ldots+d_{n-l}=d \\
d_{0}, \ldots, d_{n-l} \geq 0}} \frac{c_{0}\left(d_{0}\right) \ldots c_{n-l}\left(d_{n-l}\right)}{\left(w+d_{1}+\ldots+d_{n-l}\right)\left(w+d_{2}+\ldots+d_{n-l}\right) \ldots\left(w+d_{n-l}\right)}
\end{aligned}
$$

for all $d \geq 0$, where $c_{p}(d)$ is the coefficient of $q^{d}$ in $I_{p}(q)$. This identity is equivalent to

$$
\begin{aligned}
\sum_{p=0}^{n-l} & \frac{c_{p}(d)}{w^{n-l-p}(w+d)^{p}} \\
\quad= & \frac{\prod_{k=1}^{l} \prod_{r=0}^{a_{k} d}\left(a_{k} w+r\right)}{\langle\mathbf{a}\rangle \prod_{r=0}^{d}(w+r)^{n}}-\sum_{\substack{d_{0}+\ldots+d_{n-l}=d \\
0 \leq d_{0}, \ldots, d_{n-l}<d}} \frac{c_{0}\left(d_{0}\right) \ldots c_{n-l}\left(d_{n-l}\right)}{\left(w+d_{1}+\ldots+d_{n-l}\right) \ldots\left(w+d_{n-l}\right)} .
\end{aligned}
$$

We will use this identity to show by induction that

$$
c_{p}(d)=c_{n-l-p}(d) \quad \forall p=0,1, \ldots, n-l,
$$

thus establishing (4.8). Since $c_{p}(0)=I_{p}(0)=1$ for all $p$, (4.25) holds for $d=0$. Suppose $d \geq 1$ and (4.25) holds with $d$ replaced by every $d^{\prime}<d$. The substitution $w \rightarrow-w-d$ acts by $(-1)^{n-l}$ on the first term on the right-hand side of (4.24). It acts in the same way on the second term by the induction assumption; this can be seen by the renumbering

$$
\left(d_{0}, \ldots, d_{n-l}\right) \longrightarrow\left(d_{n-l}, \ldots, d_{0}\right) .
$$

Thus, the substitution $w \rightarrow-w-d$ acts by $(-1)^{n-l}$ on the left-hand side of (4.24), and so $c_{p}(d)=c_{n-l-p}(d)$ for all $0 \leq p \leq n-l$, as needed for the inductive step.

4.2. Proof of equation (4.5). By Lemmas 4.1 and 4.2. the functions $\mathcal{F}_{p}(w, q) \equiv \mathbf{M}^{p} \mathcal{F}(w, q)$ admit asymptotic expansions

$$
\mathcal{F}_{p}(w, q) \sim \mathrm{e}^{\mu(q) w} \sum_{s=0}^{\infty} \Phi_{p, s}(q) w^{-s} \quad(w \rightarrow \infty),
$$


with the same function $\mu(q)$ in the exponent for all $p$. Since $\mathcal{F}_{0}=\mathcal{F}$ and $\mathcal{F}_{p+1}=\mathbf{M} \mathcal{F}_{p}$

$$
\Phi_{0, s}=\Phi_{s}, \quad \Phi_{p+1, s}=\frac{1+D \mu}{I_{p}} \Phi_{p, s}+ \begin{cases}D\left(\frac{\Phi_{p, s-1}}{I_{p}}\right), & \text { if } s \geq 1, \\ 0, & \text { if } s=0 .\end{cases}
$$

Taking $s=0$ in (4.27), we find by induction that

$$
\Phi_{p, 0}=\frac{(1+D \mu)^{p}}{I_{0} I_{1} \ldots I_{p-1}} \Phi_{0} .
$$

Since $\mathcal{F}_{n}=\mathcal{F}_{0}$ by Lemma 4.1 and $\Phi_{0}(0)=1$, setting $p=n$ in the above identity we obtain

$$
(1+D \mu)^{n}=I_{0} \ldots I_{n-1} .
$$

The first claim in (4.5) now follows from (4.7) and (4.9).

For each $p \geq 0$, let

$$
H_{p}(q) \equiv \frac{L^{p}(q)}{I_{0}(q) \ldots I_{p-1}(q)} .
$$

By definition, (4.7), (4.9), (4.10), (4.28), and the first identity in (4.5),

$$
H_{0}=H_{n}=1, \quad H_{1} H_{2} \ldots H_{n}=L^{-\frac{n(l-1)}{2}}, \quad \Phi_{p, 0}=H_{p} \Phi_{0} .
$$

Taking $s=1$ in (4.27) and using the first and last equations above, we find inductively that

$$
\Phi_{p, 1}=H_{p}\left(\Phi_{1}+p \frac{D \Phi_{0}}{L}-p \frac{\Phi_{0} D L}{L^{2}}+\frac{\Phi_{0}}{L} \sum_{r=1}^{p} \frac{D H_{r}}{H_{r}}\right) \quad \forall p \geq 0 .
$$

Setting $p=n$ in this relation and using $\Phi_{n, 1}=\Phi_{1}$, along with the first and second equations in (4.29), we find that

$$
\frac{D \Phi_{0}}{\Phi_{0}}=\frac{l+1}{2} \frac{D L}{L} .
$$

Since $\Phi_{0}(0)=1=L(0)$, this confirms the second claim in (4.5).

4.3. Proof of equation (4.6). The argument in Section 4.2 can be systematized as in [ZaZ to obtain an algorithm for computing every $\Phi_{s}$ by a differential recursion.

Define $\xi_{s} \in \mathbb{Q}$ by

$$
\prod_{k=1}^{l} \prod_{j=1}^{a_{k}}\left(a_{k} D+j\right) \equiv \mathbf{a}^{\mathbf{a}} \sum_{s=0}^{n} \xi_{s} D^{s} \in \mathbb{Z}[D] ;
$$

thus, $\xi_{n}=1, \xi_{n-1}=(n+l) / 2$, and

$$
\begin{aligned}
\xi_{n-2} & =\frac{1}{24} \sum_{k=1}^{l} \frac{\left(a_{k}-1\right)\left(a_{k}+1\right)\left(3 a_{k}+2\right)}{a_{k}}+\frac{1}{4} \sum_{1 \leq i<j \leq l}\left(1+a_{i}\right)\left(1+a_{j}\right) \\
& =-\frac{1}{12} \sum_{r=1}^{l} \frac{1}{a_{r}}+\frac{3 n^{2}+n(6 l-4)+3 l^{2}-6 l}{24} .
\end{aligned}
$$


Let

$$
\widetilde{D}_{w} \equiv D+L w: \mathbb{Q}(w)[[q]] \longrightarrow \mathbb{Q}(w)[[q]] .
$$

The series $\overline{\mathcal{F}}(w, q) \equiv \mathrm{e}^{-\mu(q) w} \mathcal{F}(w, q)$ admits an asymptotic expansion

$$
\overline{\mathcal{F}}(w, q) \sim \sum_{s=0}^{\infty} \Phi_{s}(q) w^{-s} \quad(w \rightarrow \infty) .
$$

Since $1+D \mu=L$ by the first claim in (4.5) and $\mathcal{F}(w, q)$ satisfies the ordinary differential equation

$$
\left\{D_{w}^{n}-w^{n}-q \prod_{k=1}^{l} \prod_{r=1}^{a_{k}}\left(a_{k} D_{w}+r\right)\right\} \mathcal{F}=0,
$$

the series $\overline{\mathcal{F}}(w, q)$ satisfies the ordinary differential equation

$$
\mathfrak{L} \overline{\mathcal{F}}=0,
$$

where $\mathfrak{L}$ is the differential operator

$$
\begin{array}{r}
\mathfrak{L} \equiv L^{n}\left[\widetilde{D}_{w}^{n}-w^{n}-q \prod_{k=1}^{l} \prod_{r=1}^{a_{k}}\left(a_{k} \widetilde{D}_{w}+r\right)\right] \\
=\widetilde{D}_{w}^{n}-(L w)^{n}-\left(L^{n}-1\right) \sum_{s=0}^{n-1} \xi_{s} \widetilde{D}_{w}^{s} .
\end{array}
$$

Since $D L=L\left(L^{n}-1\right) / n$, as in [ZaZ, Section 2.4],

$$
\widetilde{D}_{w}^{s}=\sum_{k=0}^{s} \sum_{i=0}^{k}\left(\begin{array}{l}
s \\
i
\end{array}\right) \mathcal{H}_{s-i, k-i}\left(L^{n}\right)(L w)^{s-k} D^{i},
$$

where the polynomials $\mathcal{H}_{m, j} \in \mathbb{Q}[X]$ are defined by

$$
\begin{aligned}
\mathcal{H}_{m, j} \equiv 0 \quad \text { if } m<0, \text { or } j<0, \text { or } j>m, \quad \mathcal{H}_{0,0} \equiv 1 ; \\
\mathcal{H}_{m, j}(X) \equiv \mathcal{H}_{m-1, j}(X)+(X-1)\left(X \frac{\mathrm{d}}{\mathrm{d} X}+\frac{m-j}{n}\right) \mathcal{H}_{m-1, j-1}(X) \\
\quad \text { if } m \geq 1,0 \leq j \leq m .
\end{aligned}
$$

In particular, for $m \geq 0$

$$
\begin{aligned}
& \mathcal{H}_{m, 0}(X)=1, \quad \mathcal{H}_{m, 1}(X)=\frac{1}{n}\left(\begin{array}{c}
m \\
2
\end{array}\right)(X-1), \\
& \mathcal{H}_{m, 2}(X)=\frac{1}{n^{2}}\left(\begin{array}{c}
m \\
3
\end{array}\right)((n+1) X-1)(X-1)+\frac{3}{n^{2}}\left(\begin{array}{c}
m \\
4
\end{array}\right)(X-1)^{2} .
\end{aligned}
$$

By (4.33) and (4.34),

$$
\mathfrak{L}=\sum_{k=1}^{n}(L w)^{n-k} \mathfrak{L}_{k},
$$


where $\mathfrak{L}_{k}$ is the differential operator of order $k$ given by

$$
\mathfrak{L}_{k}=\sum_{i=0}^{k}\left[\left(\begin{array}{c}
n \\
i
\end{array}\right) \mathcal{H}_{n-i, k-i}\left(L^{n}\right)-\left(L^{n}-1\right) \sum_{r=1}^{k-i}\left(\begin{array}{c}
n-r \\
i
\end{array}\right) \xi_{n-r} \mathcal{H}_{n-i-r, k-i-r}\left(L^{n}\right)\right] D^{i} .
$$

By (4.35), the first two of these operators are

$$
\begin{aligned}
& \mathfrak{L}_{1}= n D-\frac{l+1}{2}\left(L^{n}-1\right)=n L^{\frac{l+1}{2}} D L^{-\frac{l+1}{2}}, \\
& \mathfrak{L}_{2}=\left(\begin{array}{c}
n \\
2
\end{array}\right) D^{2}-\frac{(l+2)(n-1)}{2}\left(L^{n}-1\right) D \\
& \quad+\left[\frac{(n-1)(n-2)(n-6 l-5)}{24 n} L^{n}+\frac{(3 n+6 l+5)(n-1)(n-2)}{24 n}-\xi_{n-2}\right]\left(L^{n}-1\right) .
\end{aligned}
$$

Combining (4.31), (4.32), and (4.36), we obtain the following.

Proposition 4.6. The power series $\Phi_{s} \in \mathbb{Q}[[q]], s \geq 0$, defined by (4.3), are determined by the first-order ordinary differential equations

$$
\mathfrak{L}_{1}\left(\Phi_{s}\right)+\frac{1}{L} \mathfrak{L}_{2}\left(\Phi_{s-1}\right)+\frac{1}{L^{2}} \mathfrak{L}_{3}\left(\Phi_{s-2}\right)+\ldots+\frac{1}{L^{n-1}} \mathfrak{L}_{n}\left(\Phi_{s+1-n}\right)=0, \quad s \geq 0,
$$

together with the initial conditions $\Phi_{s}(0)=\delta_{0, s}$ and $\Phi_{s}=0$ for $s<0$.

The $s=0$ case of (4.39) immediately recovers the second claim in (4.5). The $s=1$ case of (4.39) then gives

$$
n D\left(\Phi_{1} / \Phi_{0}\right)=-\frac{1}{L}\left(L^{n}-1\right)\left(\frac{n^{2}-n-3 l^{2}+1}{24 n}(n-1) L^{n}+\left[\frac{1}{12} \sum_{r=1}^{l} \frac{1}{a_{r}}-\frac{3 l^{2}-1}{24 n}\right]\right) .
$$

Along with $D L=L\left(L^{n}-1\right) / n$ and $\Phi_{1}(0)=0$, this identity gives (4.6).

\section{Computation of Reduced genus 1 GW invariants}

In this section, we deduce Theorem 3 below from Proposition 3.1, using Lemmas 5.1 and 5.3 and the properties of the hypergeometric series $\mathcal{F}(w, q)$ described by Propositions 4.3 and 4.4 . Lemma 5.1 is used to drop purely equivariant terms from the power series $\mathfrak{X}$, while Lemma 5.3 provides the relevant information about the genus 0 generating functions $\mathcal{Z}_{i}^{*}, \mathcal{Z}_{j i}^{*}$, and $\widetilde{\mathcal{Z}}_{i i}^{*}$.

Theorem 3. The generating function $\mathfrak{X}_{0}(Q)$ defined by (3.8) is given by

$$
\mathfrak{X}_{0}(Q)=Q \frac{\mathrm{d}}{\mathrm{d} Q}(\tilde{A}(q)+\tilde{B}(q))
$$


where $Q$ and $q$ are related by the mirror map (1.6) and

$$
\begin{aligned}
\tilde{A}(q)= & \frac{n}{48}\left(n-1-2 \sum_{k=1}^{l} \frac{1}{a_{k}}\right) \mu(q) \\
& -\left\{\begin{array}{l}
\frac{n+1}{48} \log \left(1-\mathbf{a}^{\mathbf{a}} q\right)+\sum_{p=0}^{\frac{n-2-l}{2}} \frac{(n-l-2 p)^{2}}{8} \log I_{p}(q), \quad \text { if } 2 \mid(n-l) ; \\
\frac{n-2}{48} \log \left(1-\mathbf{a}^{\mathbf{a}} q\right)+\sum_{p=0}^{\frac{n-3-l}{2}} \frac{(n-l-2 p)^{2}-1}{8} \log I_{p}(q), \quad \text { if } 2 \chi(n-l) ;
\end{array}\right. \\
\tilde{B}(q)= & -\frac{n}{48}\left(n-1-2 \sum_{k=1}^{l} \frac{1}{a_{k}}\right) \mu(q)+\frac{\langle\mathbf{a}\rangle}{24} \varepsilon_{0}(\mathbf{a})\left[\log I_{0}(q)\right]+\frac{\langle\mathbf{a}\rangle}{24} \varepsilon_{1}(\mathbf{a}) J(q) \\
& +\frac{l+1}{48} \log \left(1-\mathbf{a}^{\mathbf{a}} q\right)+\frac{\langle\mathbf{a}\rangle}{24} \sum_{p=2}^{n-1-l} \llbracket \prod_{\frac{(1+w)^{n}}{l}\left(1+a_{k} w\right)} \|_{w ; n-1-l-p} \llbracket \log \tilde{\mathcal{F}}(w, q) \rrbracket_{w ; p},
\end{aligned}
$$

where $\tilde{\mathcal{F}}, I_{p}, J, \mu$, and $\llbracket \cdot \rrbracket_{w ; p}$ are defined by (1.2), (1.4), (1.7), (4.3) and (2.3), respectively.

We will show that the terms $\mathcal{A}_{i}$ and $\tilde{\mathcal{A}}_{i j}$, with $j \in[n]$, in (3.16) together contribute $\frac{1}{2} Q \frac{\mathrm{d}}{\mathrm{d} Q} \mathbb{A}(q)$ to $\mathfrak{X}_{0}(Q)$, where

$$
\begin{array}{r}
\mathbb{A}(q)=\frac{n}{24}\left(n-1-2 \sum_{r=1}^{l} \frac{1}{a_{r}}\right) \mu(q)-\frac{3(n-1-l)^{2}+(n-2)}{24} \log \left(1-\mathbf{a}^{\mathbf{a}} q\right) \\
-\sum_{p=0}^{n-2-l}\left(\begin{array}{c}
n-l-p \\
2
\end{array}\right) \log I_{p}(q),
\end{array}
$$

while the terms $\mathcal{B}_{i}$ and $\tilde{\mathcal{B}}_{i j}$, with $j \in[n]$, together contribute $Q \frac{\mathrm{d}}{\mathrm{d} Q} \tilde{B}(q)$. Since $I_{p}=I_{n-l-p}$ by (4.8) and since

$$
\begin{array}{rlrl}
\sum_{r=0}^{\frac{n-2-l}{2}} \log I_{r}(q)+\frac{1}{2} \log I_{\frac{n-l}{2}}(q) & =-\frac{1}{2} \log \left(1-\mathbf{a}^{\mathbf{a}} q\right), & & \text { if } 2 \mid(n-l), \\
\sum_{r=0}^{\frac{n-1-l}{2}} \log I_{r}(q) & =-\frac{1}{2} \log \left(1-\mathbf{a}^{\mathbf{a}} q\right), & \text { if } 2 \chi(n-l),
\end{array}
$$

by (4.8) and (4.9), the expression on the right-hand side of (5.1) equals twice the right-hand side in the first equation in Theorem 3. See also (5.12) and (5.13).

5.1. Some algebraic notation and observations. This section recalls the statement of [Z5. Lemma 3.3], which shows that most terms appearing in the computation of $\mathfrak{X}(\alpha, x, Q)$ have no effect on $\mathfrak{X}_{0}(Q)$. We then set up additional related notation and make a few algebraic observations that help streamline computations in the remainder of the paper.

For each $p \in[n]$, let $\sigma_{p}$ be the $p$-th elementary symmetric polynomial in $\alpha_{1}, \ldots, \alpha_{n}$. Denote by

$$
\mathbb{Q}[\alpha]^{S_{n}} \equiv \mathbb{Q}\left[\alpha_{1}, \ldots, \alpha_{n}\right]^{S_{n}} \subset \mathbb{Q}\left[\alpha_{1}, \ldots, \alpha_{n}\right]
$$


the subspace of symmetric polynomials, by $\mathcal{I} \subset \mathbb{Q}[\alpha]^{S_{n}}$ the ideal generated by $\sigma_{1}, \ldots, \sigma_{n-1}$, and by

$$
\tilde{\mathbb{Q}}[\alpha]^{S_{n}} \equiv \mathbb{Q}\left[\alpha_{1}, \ldots, \alpha_{n}\right]_{\left\langle\alpha_{j},\left(\alpha_{j}-\alpha_{k}\right) \mid j \neq k\right\rangle}^{S_{S_{n}}} \subset \mathbb{Q}_{\alpha}
$$

the subalgebra of symmetric rational functions in $\alpha_{1}, \ldots, \alpha_{n}$ whose denominators are products of $\alpha_{j}$ and $\left(\alpha_{j}-\alpha_{k}\right)$ with $j \neq k$. For each $i=1, \ldots, n$, let

$$
\tilde{\mathbb{Q}}_{i}[\alpha]^{S_{n-1}} \equiv \mathbb{Q}\left[\alpha_{1}, \ldots, \alpha_{n}\right]_{\left\langle\alpha_{i},\left(\alpha_{i}-\alpha_{k}\right) \mid k \neq i\right\rangle}^{S_{n-1}} \subset \mathbb{Q}_{\alpha}
$$

be the subalgebra consisting of rational functions symmetric in $\left\{\alpha_{k}: k \neq i\right\}$ and with denominators that are products of $\alpha_{i}$ and $\left(\alpha_{i}-\alpha_{k}\right)$ with $k \neq i$. Let

$$
\mathcal{K}_{i} \equiv \operatorname{Span}_{\mathbb{Q}}\left\{\mathcal{I} \cdot \tilde{\mathbb{Q}}_{i}[\alpha]^{S_{n-1}}, \alpha_{i}^{n-2} \cdot \mathcal{I} \tilde{\mathbb{Q}}[\alpha]^{S_{n}},\left\{1, \alpha_{i}, \ldots, \alpha_{i}^{n-3}, \alpha_{i}^{n-1}\right\} \cdot \tilde{\mathbb{Q}}[\alpha]^{S_{n}}\right\}
$$

Lemma 5.1 ([Z5, Lemma 3.3]). 8 If $n \geq 2$, the linear span of $\alpha_{i}^{n-2}$ is disjoint from $\mathcal{K}_{i}:$

$$
\operatorname{Span}\left\{\alpha_{i}^{n-2}\right\} \cap \mathcal{K}_{i}=\{0\} \subset \mathbb{Q}_{\alpha} .
$$

For each $i=1, \ldots, n$, let

$$
\tilde{\mathbb{Q}}_{i}[\alpha]_{\hbar}^{S_{n-1}} \equiv \mathbb{Q}\left(\hbar, \alpha_{i}\right)[\alpha]_{\left\langle\left(\alpha_{i}-\alpha_{k}+r \hbar\right) \mid k \in i, r \in \mathbb{Z}, k \neq i\right\rangle}^{S_{n-1}} \subset \mathbb{Q}_{\alpha}(\hbar)
$$

be the subalgebra consisting of rational functions symmetric in $\left\{\alpha_{k}: k \neq i\right\}$ and with denominators that are a product of a polynomial with rational coefficients in $\hbar$ and $\alpha_{i}$ and of linear factors of the form $\left(\alpha_{i}-\alpha_{k}+r \hbar\right), r \in \mathbb{Z}$. Denote by

$$
\mathcal{S}_{i, \hbar} \subset \tilde{\mathbb{Q}}_{i}[\alpha]_{\hbar}^{S_{n-1}}
$$

the subalgebra consisting of rational functions of the form $A+B \prod_{k=1}^{l}\left(a_{k} \alpha_{i}+\hbar\right)$ with $A, B \in \tilde{\mathbb{Q}}_{i}[\alpha]_{\hbar}^{S_{n-1}}$ both regular at $\hbar=-a_{k} \alpha_{i}$ for every $k \in[l]$ and the denominator of $A$ an element of $\mathbb{Q}\left[\alpha_{i}, \hbar\right]$. We define

$$
\tilde{\mathbb{Q}}_{i}[\alpha]_{\hbar_{1}, \hbar_{2}}^{S_{n-1}} \subset \mathbb{Q}_{\alpha}\left(\hbar_{1}, \hbar_{2}\right)
$$

to be the subalgebra generated by $\tilde{\mathbb{Q}}_{i}[\alpha]_{\hbar_{1}}^{S_{n-1}}$ and $\tilde{\mathbb{Q}}_{i}[\alpha]_{\hbar_{2}}^{S_{n-1}}$. If in addition $j \in[n]$, let

$$
\begin{aligned}
& \mathcal{K}^{(i, j)} \equiv \operatorname{Span}_{\mathbb{Q}}\left\{\alpha_{i}^{n-2} \mathcal{I} \cdot \tilde{\mathbb{Q}}_{j}[\alpha]^{S_{n-1}},\left\{1, \alpha_{i}, \ldots, \alpha_{i}^{n-3}, \alpha_{i}^{n-1}\right\} \tilde{\mathbb{Q}}_{j}[\alpha]^{S_{n-1}}\right\} \subset \mathbb{Q}_{\alpha}, \\
& \mathcal{K}_{\hbar}^{(i, j)} \equiv \operatorname{Span}_{\mathbb{Q}}\left\{\alpha_{i}^{n-2} \mathcal{I} \cdot \mathcal{S}_{j, \hbar},\left\{1, \alpha_{i}, \ldots, \alpha_{i}^{n-3}, \alpha_{i}^{n-1}\right\} \mathcal{S}_{j, \hbar}\right\} \subset \mathbb{Q}_{\alpha}(\hbar) .
\end{aligned}
$$

All statements in the next lemma follow immediately from the definitions.

\footnotetext{
${ }^{8}$ The definition of $\mathcal{K}_{i}$ in [Z5] is missing $\alpha_{i}^{n-2} \mathcal{I} \tilde{\mathbb{Q}}[\alpha]^{S_{n}}$, but the proof of [Z5, Lemma 3.3] still goes through. This change adds the term $\alpha_{i}^{n-1} g_{n-1}$, with $g_{n-1} \in \mathcal{I}$, to the second numerator in [Z5 (3.13)] and $g_{n-1}$ to the right-hand side of [Z5 (3.15)]. As $g_{n-1} \in \mathcal{I}$, this addition has no effect on the concluding sentence in the proof of Lemma 3.3 in [Z5].
} 
Lemma 5.2. If $i \in[n]$, then

$$
\begin{aligned}
& F \in \tilde{\mathbb{Q}}_{i}[\alpha]_{\hbar}^{S_{n-1}}[[q]] \quad \Longrightarrow \quad \Re_{\hbar=0} F, \mathfrak{R}_{\hbar=\infty} F \in \tilde{\mathbb{Q}}_{i}[\alpha]^{S_{n-1}}[[q]] ; \\
& F \in \tilde{\mathbb{Q}}_{i}[\alpha]_{\hbar_{1}, \hbar_{2}}^{S_{n-1}}[[q]] \quad \Longrightarrow \quad \Re_{\hbar_{1}=0} \Re_{\hbar_{2}=0}\left\{\frac{F}{\hbar_{1}+\hbar_{2}}\right\} \in \tilde{\mathbb{Q}}_{i}[\alpha]^{S_{n-1}}[[q]] ; \\
& F \in \mathcal{S}_{i, \hbar}[[q]] \quad \Longrightarrow \quad \mathfrak{R}_{\hbar=-\mathbf{a} \alpha_{i}}\left\{\frac{F}{\prod_{r=1}^{l}\left(a_{r} \alpha_{i}+\hbar\right)}\right\} \in \tilde{\mathbb{Q}}_{i}[\alpha]^{S_{n-1}}[[q]] .
\end{aligned}
$$

If in addition $F, G \in q \tilde{\mathbb{Q}}_{i}[\alpha]_{\hbar}^{S_{n-1}}[[q]]$, then

$F-G \in \mathcal{I} \cdot \tilde{\mathbb{Q}}_{i}[\alpha]_{\hbar}^{S_{n-1}}[[q]] \Longrightarrow \mathrm{e}^{F}-\mathrm{e}^{G}, \log (1+F)-\log (1+G) \in \mathcal{I} \cdot q \tilde{\mathbb{Q}}_{i}[\alpha]_{\hbar}^{S_{n-1}}[[q]]$.

5.2. The genus 0 generating functions. We will now express the genus 0 generating functions $\mathcal{Z}_{i}^{*}, \mathcal{Z}_{i j}^{*}$, and $\widetilde{\mathcal{Z}}_{i i}^{*}$ defined in Section 3.3 in terms of the hypergeometric series $\mathcal{F}$ of (4.1) and the operator $\mathbf{M}$ of (1.3).

Lemma 5.3. The genus 0 generating functions $\mathcal{Z}_{i}^{*}, \mathcal{Z}_{i j}^{*}$, and $\widetilde{\mathcal{Z}}_{i i}^{*}$ satisfy

$$
\begin{gathered}
{\left[\left(\alpha_{i}+\hbar\right)^{n}-\alpha_{i}^{n}\right]\left[1+\mathcal{Z}_{i}^{*}(\hbar, Q)-\mathrm{e}^{-J(q) \frac{\alpha_{i}}{\hbar}} \frac{\mathcal{F}\left(\alpha_{i} / \hbar, q\right)}{I_{0}(q)}\right] \in \mathcal{I} \cdot q \mathcal{S}_{i, \hbar}[[q]],} \\
{\left[\left(\alpha_{j}+\hbar\right)^{n}-\alpha_{j}^{n}\right]\left[\alpha_{i}^{n-2} \alpha_{j}+\hbar \mathcal{Z}_{j i}^{*}(\hbar, Q)-\alpha_{i}^{n-2} \alpha_{j} \mathrm{e}^{-J(q) \frac{\alpha_{j}}{\hbar}} \frac{\mathbf{M} \mathcal{F}\left(\alpha_{j} / \hbar, q\right)}{I_{1}(q)}\right]} \\
\quad \in \mathcal{K}_{\hbar}^{(i, j)}[[q]] 9 \\
n \alpha_{i}^{n-1}+2\left(\hbar_{1}+\hbar_{2}\right) \hbar_{1} \hbar_{2} \widetilde{\mathcal{Z}}_{i i}^{*}\left(\hbar_{1}, \hbar_{2}, Q\right) \\
-\alpha_{i}^{n-1} \mathrm{e}^{-J(q) \alpha_{i}\left(\frac{1}{h_{1}}+\frac{1}{\hbar_{2}}\right)} \mathbb{F}\left(\alpha_{i} / \hbar_{1}, \alpha_{i} / \hbar_{2}, q\right) \in \mathcal{I} \cdot \tilde{\mathbb{Q}}_{i}[\alpha]_{\hbar_{1}, \hbar_{2}}^{S_{n-1}}[[q]],
\end{gathered}
$$

where

$$
\begin{aligned}
\mathbb{F}\left(w_{1}, w_{2}, q\right)= & \sum_{p=0}^{n-1-l} \frac{\mathbf{M}^{p} \mathcal{F}\left(w_{1}, q\right)}{I_{p}(q)} \frac{\mathbf{M}^{n-1-l-p} \mathcal{F}\left(w_{2}, q\right)}{I_{n-1-l-p}(q)} \\
& +\sum_{p=1}^{l} \frac{\mathbf{M}^{n-1-l+p} \mathcal{F}\left(w_{1}, q\right)}{I_{n-1-l+p}(q)} \frac{\mathbf{M}^{n-p} \mathcal{F}\left(w_{2}, q\right)}{I_{n-p}(q)}
\end{aligned}
$$

and $Q$ and $q$ are related by the mirror map (1.6).

Proof. By [Gi, Theorem 11.8],

$$
1+\mathcal{Z}_{i}^{*}(\hbar, Q)=\mathrm{e}^{\frac{-J(q) \alpha_{i}+C(q) \sigma_{1}}{\hbar}} \frac{\mathbb{Y}\left(\hbar, \alpha_{i}, q\right)}{I_{0}(q)}
$$

${ }^{9}$ Note that (5.4) implies that

$$
\begin{aligned}
& \alpha_{i}^{n-2} \alpha_{j}+\hbar \mathcal{Z}_{j i}^{*}(\hbar, Q)-\alpha_{i}^{n-2} \alpha_{j} \mathrm{e}^{-J(q) \frac{\alpha_{j}}{\hbar}} \frac{\mathbf{M} \mathcal{F}\left(\alpha_{j} / \hbar, q\right)}{I_{1}(q)} \\
& \quad \in \operatorname{Span}_{\mathbb{Q}}\left\{\alpha_{i}^{n-2} \mathcal{I} \cdot \tilde{\mathbb{Q}}_{j}[\alpha]_{\hbar}^{S_{n-1}},\left\{1, \alpha_{i}, \ldots, \alpha_{i}^{n-3}, \alpha_{i}^{n-1}\right\} \tilde{\mathbb{Q}}_{j}[\alpha]_{\hbar}^{S_{n-1}}\right\}[[q]] ;
\end{aligned}
$$

this is the only information about $\mathcal{Z}_{j i}^{*}(Q)$ used in computing the nonequivariant part of $\tilde{\mathcal{A}}_{i j}(Q)$ in Section 5.3 
for some $C \in q \mathbb{Q}[[q]]$ and

$$
\mathbb{Y}(\hbar, x, q) \equiv \sum_{d=0}^{\infty} q^{d} \frac{\prod_{k=1}^{l} \prod_{r=1}^{a_{k} d}\left(a_{k} x+r \hbar\right)}{\prod_{r=1}^{d}\left[\prod_{k=1}^{n}\left(x-\alpha_{k}+r \hbar\right)-\prod_{k=1}^{n}\left(x-\alpha_{k}\right)\right]} .
$$

There is no term $\prod_{k=1}^{n}\left(x-\alpha_{k}\right)$ in the generating function used in place of $\mathbb{Y}$ in Gi], but putting it does not affect the validity of [Gi, Theorem 11.8] as it vanishes under all evaluations $x \longrightarrow \alpha_{i}$. On the other hand, with this extra term in place, $\mathbb{Y}$ becomes a function of $\hbar, x, \sigma_{1}, \ldots, \sigma_{n-1}$, and not $\sigma_{n}$. Since all denominators in $\mathbb{Y}\left(\hbar, \alpha_{i}, q\right)$ are products of $\alpha_{i}-\alpha_{k}+r \hbar$ with $r \in \mathbb{Z}$,

$$
\mathbb{Y}\left(\hbar, \alpha_{i}, q\right)-\mathcal{F}\left(\alpha_{i} / \hbar, q\right) \in \mathcal{I} \cdot \tilde{\mathbb{Q}}_{i}[\alpha]_{\hbar}^{S_{n-1}}[[q]] .
$$

If $a_{k} \neq 1,2$ or $n$ is odd, the denominators in the above expression do not vanish at $\hbar=-a_{k} \alpha_{i}$, and so the difference lies in $\mathcal{I} \cdot q \mathcal{S}_{i, \hbar}[[q]]$. Otherwise, the denominators have a simple zero at $\hbar=-a_{k} \alpha_{i}$ (in $q$-degree at least 2 if $a_{k}=1$ ). If $a_{k}=2$ and $n$ is even, the factor $\left[\left(\alpha_{i}+\hbar\right)^{n}-\alpha_{i}^{n}\right]$ has a zero at $\hbar=-a_{k} \alpha_{i}$ as well, and so

$$
\left[\left(\alpha_{i}+\hbar\right)^{n}-\alpha_{i}^{n}\right]\left[\mathbb{Y}\left(\hbar, \alpha_{i}, q\right)-\mathcal{F}\left(\alpha_{i} / \hbar, q\right)\right] \in \mathcal{I} \cdot q \mathcal{S}_{i, \hbar}[[q]] .
$$

The case $a_{k}=1$ is excluded by the assumption on a in Section 1. Thus, (5.3) follows from (5.6).

By (3.2), [PoZ, Theorem 6], and the same reasoning as in the previous paragraph,

$$
\sum_{\substack{p+r+s=n-1 \\
p, r, s \geq 0}}(-1)^{s} \sigma_{s} \alpha_{i}^{p} \alpha_{j}^{r}+\hbar \mathcal{Z}_{j i}^{*}(\hbar, Q)=\mathrm{e}^{\frac{-J(q) \alpha_{j}+C(q) \sigma_{1}}{\hbar}} \sum_{\begin{array}{c}
p+r+s=n-1 \\
p, r, s \geq 0
\end{array}}(-1)^{s} \sigma_{s} \alpha_{i}^{p_{i}} \mathbb{Y}_{r}\left(\hbar, \alpha_{j}, q\right),
$$

where $\mathbb{Y}_{r}(h, x, q) \in \mathbb{Q}_{\alpha}(h, x)[[q]]$ is a power series such that

$$
\begin{aligned}
& \mathbb{Y}_{r}\left(\hbar, \alpha_{j}, q\right) \in \mathcal{S}_{j, \hbar}[[q]] \quad \text { and } \\
& {\left[\left(\alpha_{j}+\hbar\right)^{n}-\alpha_{j}^{n}\right]\left[\mathbb{Y}_{r}\left(\hbar, \alpha_{j}, q\right)-\alpha_{j}^{r} \frac{\mathbf{M}^{r} \mathcal{F}\left(\alpha_{j} / \hbar, q\right)}{I_{r}(q)}\right] \in \mathcal{I} \cdot q \mathcal{S}_{j, \hbar}[[q]] .10 }
\end{aligned}
$$

The claim (5.4) thus follows from (5.7).

Finally, by [PoZ, Theorem 6] and [PoZ, (3.5)],

$$
\begin{aligned}
& \sum_{\substack{p+r+s=n-1 \\
p, r, s \geq 0}}(-1)^{s} \sigma_{s} \alpha_{i}^{p+r}+2\left(\hbar_{1}+\hbar_{2}\right) \hbar_{1} \hbar_{2} \widetilde{\mathcal{Z}}_{i i}^{*}\left(\hbar_{1}, \hbar_{2}, Q\right) \\
& =\mathrm{e}^{\left(-J(q) \alpha_{i}+C(q) \sigma_{1}\right)\left(\frac{1}{\hbar_{1}}+\frac{1}{\hbar_{2}}\right)} \sum_{\substack{\alpha_{i} \\
p+r+s=n-1 \\
p, r, s \geq 0}}^{l}(-1)^{s} \sigma_{s} \mathbb{Y}_{p}\left(\hbar_{1}, \alpha_{i}, q\right) \mathbb{Y}_{r-l}\left(\hbar_{2}, \alpha_{i}, q\right), \\
& (5.10) \quad \sum_{\substack{s_{1}+s_{2}=k \\
0 \leq s_{1} \leq n}}(-1)^{s_{1}} \sigma_{s_{1}} \mathbb{Y}_{s_{2}}\left(\hbar, \alpha_{i}, q\right)=0 \quad \text { if } \quad n-l \leq k \leq n-1,
\end{aligned}
$$

\footnotetext{
${ }^{10}$ In the notation of $\underline{\mathrm{PoZ}}, \mathbb{Y}_{r}(\hbar, x, q)=\mathcal{Y}_{r}(\hbar, x, q) / x^{l}$
} 
where $\mathbb{Y}_{r}(h, x, q) \in \mathbb{Q}_{\alpha}(h, x)[[q]]$ is a power series such that

$$
\mathbb{Y}_{r}\left(\hbar, \alpha_{i}, q\right) \in \tilde{\mathbb{Q}}_{i}[\alpha]_{\hbar}^{S_{n-1}}[[q]] .
$$

By (5.10) and (5.11),

$$
\mathbb{Y}_{-p}\left(\hbar, \alpha_{i}, q\right)-\alpha_{i}^{-n} \mathbb{Y}_{n-p}\left(\hbar, \alpha_{i}, q\right) \in \mathcal{I} \cdot \tilde{\mathbb{Q}}_{i}[\alpha]_{\hbar}^{S_{n-1}}[[q]] \quad \forall p=1,2, \ldots, l .
$$

Thus, (5.5) follows from (5.9), (5.11), and (5.8).

5.3. Proof of Theorem 3, We will use Lemmas 5.155 .3 to extract the coefficients of $\alpha_{i}^{n-2}$ from the expressions of Proposition 3.1 modulo $\mathcal{K}_{i}[[q]]$, as follows. We will show that, in the notation of Theorem 3 and Proposition [3.1.

$$
\begin{aligned}
& \mathcal{A}_{i}(Q)+\sum_{j=1}^{n} \tilde{\mathcal{A}_{i j}}(Q) \cong \alpha_{i}^{n-2} Q \frac{d \tilde{A}(q)}{d Q} \text { modulo } \mathcal{K}_{i}[[q]] \\
& \mathcal{B}_{i}(Q)+\sum_{j=1}^{n} \tilde{\mathcal{B}_{i j}}(Q) \cong \alpha_{i}^{n-2} Q \frac{d \tilde{B}(q)}{d Q} \text { modulo } \mathcal{K}_{i}[[q]]
\end{aligned}
$$

By (3.8), Proposition 3.1 and Lemma 5.1, this implies Theorem 3 ,

Let $D \equiv q \frac{\mathrm{d}}{\mathrm{d} q}$ as in Section 4 . We begin by computing residues of the transforms of $\mathcal{F}$ that appear in the description of the generating functions in Lemma 5.3 .

Lemma 5.4. With $\mu, \Phi_{0}, L, \mathbb{A} \in \mathbb{Q}[[q]]$ given by (4.3), (4.4), and (5.1),

$$
\begin{aligned}
& \mathfrak{R}_{\hbar=0}\left\{\hbar^{-1} \mathrm{e}^{-\mu(q) \frac{\alpha_{j}}{\hbar}} \mathbf{M} \mathcal{F}\left(\alpha_{j} / \hbar, q\right)\right\}=\frac{L(q) \Phi_{0}(q)}{I_{0}(q)}, \\
& \mathfrak{R}_{h_{1}=0} \mathfrak{R}_{h_{2}=0}\left\{\frac{\mathrm{e}^{-\mu(q) \alpha_{i}\left(\hbar_{1}^{-1}+\hbar_{2}^{-1}\right)}}{\hbar_{1} \hbar_{2}\left(\hbar_{1}+\hbar_{2}\right)} \mathbb{F}\left(\alpha_{i} / \hbar_{1}, \alpha_{i} / \hbar_{2}, q\right)\right\}=\alpha_{i}^{-1} L(q)^{-1} D \mathbb{A}(q) .
\end{aligned}
$$

Proof. By Lemmas 4.1 and 4.2, (4.3), (1.3), and the first statement in (4.5),

$$
\mathbf{M}^{p} \mathcal{F}(w, q) \sim \mathrm{e}^{\mu(q) w} \sum_{s=0}^{\infty} \Phi_{p, s}(q) w^{-s} \quad(w \rightarrow \infty),
$$

where

$$
\Phi_{0, s}=\Phi_{s}, \quad \Phi_{p+1, s}=\frac{L}{I_{p}} \Phi_{p, s}+ \begin{cases}D\left(\frac{\Phi_{p, s-1}}{I_{p}}\right), & \text { if } s \geq 1, \\ 0, & \text { if } s=0 .\end{cases}
$$

The $s=0,1$ cases of the recursion give

$$
\Phi_{p, 0}=H_{p} \Phi_{0}, \quad \Phi_{p, 1}=H_{p}\left(\Phi_{1}+p D\left(\frac{\Phi_{0}}{L}\right)+\frac{\Phi_{0}}{L} \sum_{r=1}^{p} \frac{D H_{r}}{H_{r}}\right),
$$

where $H_{p}=L^{p} /\left(I_{0} \ldots I_{p-1}\right)$ as in Section 4.2 The $p=1$ case of the first identity above gives (5.14).

On the other hand, for all $p, r \geq 0$

$$
\begin{aligned}
\mathfrak{R}_{h_{1}=0} \mathfrak{R}_{h_{2}=0}\left\{\frac{\mathrm{e}^{-\mu(q) \alpha_{i}\left(\hbar_{1}^{-1}+\hbar_{2}^{-1}\right)}}{\hbar_{1} \hbar_{2}\left(\hbar_{1}+\hbar_{2}\right)} \mathbf{M}^{p} \mathcal{F}\left(\alpha_{i} / \hbar_{1}, q\right) \mathbf{M}^{r} \mathcal{F}\left(\alpha_{i} / \hbar_{2}, q\right)\right\} \\
=\mathfrak{R}_{h_{1}=0}\left\{\frac{\mathrm{e}^{-\mu(q) \alpha_{i} / \hbar_{1}}}{\hbar_{1}^{2}} \mathbf{M}^{p} \mathcal{F}\left(\alpha_{i} / \hbar_{1}, q\right) \cdot \Phi_{r, 0}(q)\right\}=\alpha_{i}^{-1} \Phi_{p, 1}(q) \Phi_{r, 0}(q) .
\end{aligned}
$$


By (4.7)-(4.9),

$$
\begin{aligned}
& H_{p} H_{n-1-l-p}=\frac{I_{p} I_{n-1-l-p}}{L^{l+1}} \quad \text { if } 0 \leq p \leq n-1-l, \\
& H_{n-1-l+p} H_{n-p}=\frac{I_{n-1-l+p} I_{n-p}}{L^{l+1}} \quad \text { if } 1 \leq p \leq l .
\end{aligned}
$$

Thus, by (5.17) and the second statement in (4.5),

$$
\begin{aligned}
\mathfrak{R}_{h_{1}=0} \Re_{h_{2}=0}\left\{\frac{\mathrm{e}^{-\mu(q) \alpha_{i}\left(\hbar_{1}^{-1}+\hbar_{2}^{-1}\right)}}{\hbar_{1} \hbar_{2}\left(\hbar_{1}+\hbar_{2}\right)} \mathbb{F}\left(\alpha_{i} / \hbar_{1}, \alpha_{i} / \hbar_{2}, q\right)\right\} \\
\quad=\alpha_{i}^{-1} L^{-\frac{l+1}{2}}\left(n \Phi_{1}+\left(\begin{array}{c}
n \\
2
\end{array}\right) D\left(L^{\frac{l-1}{2}}\right)+L^{\frac{l-1}{2}} \sum_{r=1}^{n-1}(n-r) \frac{D H_{r}}{H_{r}}\right) .
\end{aligned}
$$

By (4.7), (4.9) and (4.10),

$$
\begin{aligned}
\sum_{r=1}^{n-1}(n-r) \frac{D H_{r}}{H_{r}}=\sum_{r=1}^{n-l}(n-l-r) \frac{D H_{r}}{H_{r}}+l \sum_{r=1}^{n-l} \frac{D H_{r}}{H_{r}}+\sum_{r=1}^{l-1}(l-r) \frac{D H_{n-l+r}}{H_{n-l+r}} \\
=\left(\begin{array}{c}
n-l+1 \\
3
\end{array}\right) \frac{D L}{L}-\sum_{r=1}^{n-l} \sum_{p=0}^{r-1}(n-l-r) \frac{D I_{p}}{I_{p}} \\
-\frac{(l-1) l(n-l)}{2} \frac{D L}{L}-\frac{(l-1) l(2 l-1)}{6} \frac{D L}{L} \\
=\left(\begin{array}{c}
n-l+1 \\
3
\end{array}\right) \frac{D L}{L}-\frac{(l-1) l(3 n-l-1)}{6} \frac{D L}{L}-\sum_{p=0}^{n-l}\left(\begin{array}{c}
n-l-p \\
2
\end{array}\right) \frac{D I_{p}}{I_{p}} .
\end{aligned}
$$

The second identity in the lemma follows from the last two equations along with (4.5), (4.6), $D L=L\left(L^{n}-1\right) / n$, and (4.4).

We will now compute (5.12). By Lemma 5.2, (5.3), (3.14), (3.15), and (4.3),

$$
\begin{aligned}
\eta_{i}(Q)-(\mu(q)-J(q)) \alpha_{i} & \in \mathcal{I} \cdot q \tilde{\mathbb{Q}}_{i}[\alpha]^{S_{n-1}}[[q]], \\
\Phi_{0}\left(\alpha_{i}, Q\right)-\frac{\Phi_{0}(q)}{I_{0}(q)} & \in \mathcal{I} \cdot q \tilde{\mathbb{Q}}_{i}[\alpha]^{S_{n-1}}[[q]] .
\end{aligned}
$$

Thus, by Proposition 3.1, Lemma 5.2, (5.5), and (5.15),

$$
\mathcal{A}_{i}(Q)-\alpha_{i}^{n-2} \frac{I_{0}(q) D \mathbb{A}(q)}{2 L(q) \Phi_{0}(q)} \in \mathcal{I} \cdot \tilde{\mathbb{Q}}_{i}[\alpha]^{S_{n-1}}[[q]]
$$

By Proposition 3.1, Lemma 5.2, (5.4), (5.14), (5.18) and (5.20),

$$
\begin{aligned}
& \sum_{j=1}^{n} \tilde{\mathcal{A}}_{i j}(Q)-\sum_{j=1}^{n}\left(\frac{\alpha_{j}^{n-2} I_{0}(q) D \mathbb{A}(q) /\left(2 L(q) \Phi_{0}(q)\right)}{\prod_{k \neq j}\left(\alpha_{j}-\alpha_{k}\right)}\left[\frac{L(q) \Phi_{0}(q)}{I_{0}(q) I_{1}(q)}-1\right] \alpha_{i}^{n-2} \alpha_{j}\right) \\
& \quad \in \mathcal{K}_{i}[[q]] .
\end{aligned}
$$


By the residue theorem on $S^{2}$,

$$
\sum_{j=1}^{n} \frac{\alpha_{j}^{n-1}}{\prod_{k \neq j}\left(\alpha_{j}-\alpha_{k}\right)}=\sum_{j=1}^{n} \mathfrak{R}_{z=\alpha_{j}}\left\{\frac{z^{n-1}}{\prod_{k=1}^{n}\left(z-\alpha_{k}\right)}\right\}=-\mathfrak{R}_{z=\infty}\left\{\frac{z^{n-1}}{\prod_{k=1}^{n}\left(z-\alpha_{k}\right)}\right\}=1 .
$$

Thus, by (5.20) and (5.21),

$$
\mathcal{A}_{i}(Q)+\sum_{j=1}^{n} \tilde{\mathcal{A}}_{i j}(Q)-\alpha_{i}^{n-2} \frac{D \mathbb{A}(q)}{2 I_{1}(q)} \in \mathcal{K}_{i}[[q]] .
$$

Since $\frac{1}{I_{1}(q)} D=Q \frac{\mathrm{d}}{\mathrm{d} Q}$, this proves the claim stated in the sentence after Theorem 3

We next compute (5.13). Let

$$
\tilde{\mathbb{B}}(q) \equiv-\frac{24}{\langle\mathbf{a}\rangle} \tilde{B}(q)
$$

with $\tilde{B}(q)$ as in Theorem 3 and

$$
\alpha_{j}^{n-2-l} B(q) \equiv \Re_{\hbar=0, \infty,-\mathbf{a} \alpha_{j}}\left\{\frac{\left(\alpha_{j}+\hbar\right)^{n}-\alpha_{j}^{n}}{\hbar^{3} \prod_{r=1}^{l}\left(a_{r} \alpha_{j}+\hbar\right)} \frac{\mathrm{e}^{-J(q) \frac{\alpha_{j}}{\hbar}} \mathcal{F}\left(\alpha_{j} / \hbar, q\right) / I_{0}(q)-1}{\mathrm{e}^{-J(q) \frac{\alpha_{j}}{\hbar}} \mathcal{F}\left(\alpha_{j} / \hbar, q\right) / I_{0}(q)}\right\} .
$$

Lemma 5.5. With notation as above,

$$
\begin{array}{r}
\mathfrak{R}_{\hbar=0, \infty,-\mathbf{a} \alpha_{j}}\left\{\frac{\left(\alpha_{j}+\hbar\right)^{n}-\alpha_{j}^{n}}{\hbar^{3} \prod_{r=1}^{l}\left(a_{r} \alpha_{j}+\hbar\right)} \frac{\mathrm{e}^{-J(q) \frac{\alpha_{j}}{\hbar}} \frac{\mathbf{M} \mathcal{F}\left(\alpha_{j} / \hbar, q\right)}{I_{1}(q)}-1}{\mathrm{e}^{-J(q) \frac{\alpha_{j}}{\hbar}} \mathcal{F}\left(\alpha_{j} / \hbar, q\right) / I_{0}(q)}\right\} \\
=\alpha_{j}^{n-2-l}\left(Q \frac{\mathrm{d} \tilde{\mathbb{B}}}{\mathrm{d} Q}(q)+B(q)\right)
\end{array}
$$

$$
\mathfrak{R}_{\hbar=0, \infty,-\mathbf{a} \alpha_{j}}\left\{\frac{\left(\alpha_{j}+\hbar\right)^{n}-\alpha_{j}^{n}}{\hbar^{2} \prod_{r=1}^{l}\left(a_{r} \alpha_{j}+\hbar\right)} \log \left(\mathrm{e}^{-J(q) \frac{\alpha_{j}}{\hbar}} \frac{\mathcal{F}\left(\alpha_{j} / \hbar, q\right)}{I_{0}(q)}\right)\right\}=\alpha_{j}^{n-1-l} \tilde{\mathbb{B}}(q) .
$$

Proof. Since $I_{1}(q)=1+q \frac{\mathrm{d}}{\mathrm{d} q} J(q)$ and $Q \frac{\mathrm{d}}{\mathrm{d} Q}=\frac{1}{I_{1}} q \frac{\mathrm{d}}{\mathrm{d} q}$,

$$
\begin{aligned}
\mathrm{e}^{-J(q) w} \frac{\mathbf{M} \mathcal{F}(w, q)}{I_{1}(q)} & =\frac{1}{I_{1}(q)}\left\{1+q \frac{\mathrm{d} J}{\mathrm{~d} q}(q)+\frac{q}{w} \frac{\mathrm{d}}{\mathrm{d} q}\right\}\left(\mathrm{e}^{-J(q) w} \frac{\mathcal{F}(w, q)}{I_{0}(q)}\right) \\
& =\left\{1+\frac{Q}{w} \frac{\mathrm{d}}{\mathrm{d} Q}\right\}\left(\mathrm{e}^{-J(q) w} \frac{\mathcal{F}(w, q)}{I_{0}(q)}\right) .
\end{aligned}
$$

Along with the definition of $B$ and (5.25), this gives (5.24).

By the residue theorem on $S^{2}$, the terms $\mathrm{e}^{-J(q) \alpha_{j} / \hbar}$ and $I_{0}(q)$ do not affect the left-hand side of (5.25). Since the coefficients of the positive 
powers of $q$ in $\left[\left(\alpha_{j}+\hbar\right)^{n}-\alpha_{j}^{n}\right] \mathcal{F}\left(\alpha_{j} / \hbar, q\right)$ vanish at $\hbar=-a_{k} \alpha_{j}$ to the same order as $\prod_{r=1}^{l}\left(a_{r} \alpha_{j}+\hbar\right), 11$ it follows that

$$
\begin{aligned}
\mathfrak{R}_{\hbar=0, \infty,-\mathbf{a} \alpha_{j}}\left\{\frac{\left(\alpha_{j}+\hbar\right)^{n}-\alpha_{j}^{n}}{\hbar^{2} \prod_{r=1}^{l}\left(a_{r} \alpha_{j}+\hbar\right)} \log \left(\mathrm{e}^{-J(q) \frac{\alpha_{j}}{\hbar}} \frac{\mathcal{F}\left(\alpha_{j} / \hbar, q\right)}{I_{0}(q)}\right)\right\} \\
=\Re_{\hbar=0, \infty}\left\{\frac{\left(\alpha_{j}+\hbar\right)^{n}-\alpha_{j}^{n}}{\hbar^{2} \prod_{r=1}^{l}\left(a_{r} \alpha_{j}+\hbar\right)} \log \mathcal{F}\left(\alpha_{j} / \hbar, q\right)\right\} .
\end{aligned}
$$

Expanding $\left(\alpha_{j}+\hbar\right)^{n}-\alpha_{j}^{n}$ and using (4.3), we obtain

$$
\begin{aligned}
\mathfrak{R}_{\hbar=0}\left\{\frac{\left(\alpha_{j}+\hbar\right)^{n}-\alpha_{j}^{n}}{\hbar^{2} \prod_{r=1}^{l}\left(a_{r} \alpha_{j}+\hbar\right)} \log \mathcal{F}\left(\alpha_{j} / \hbar, q\right)\right\} \\
\left.=\frac{\alpha_{j}^{n-1-l}}{\langle\mathbf{a}\rangle}\left(\left[\begin{array}{l}
n \\
2
\end{array}\right)-n \sum_{r=1}^{l} \frac{1}{a_{r}}\right] \mu(q)+n \log \Phi_{0}(q)\right) .
\end{aligned}
$$

On the other hand,

$$
\begin{gathered}
\mathfrak{R}_{\hbar=\infty}\left\{\frac{\left(\alpha_{j}+\hbar\right)^{n}-\alpha_{j}^{n}}{\hbar^{2} \prod_{r=1}^{l}\left(a_{r} \alpha_{j}+\hbar\right)} \log \mathcal{F}\left(\alpha_{j} / \hbar, q\right)\right\} \\
=-\mathfrak{R}_{w=0}\left\{\frac{\left(\alpha_{j} w+1\right)^{n}-\alpha_{j}^{n} w^{n}}{w^{n-l} \prod_{r=1}^{l}\left(1+a_{r} \alpha_{j} w\right)} \log \mathcal{F}\left(w \alpha_{j}, q\right)\right\} \\
=-\alpha_{j}^{n-1-l} \sum_{p=0}^{n-1-l} \llbracket \frac{(1+w)^{n}}{\prod_{k=1}^{l}\left(1+a_{k} w\right)} \|_{w ; n-1-l-p} \llbracket \log \mathcal{F}(w, q) \rrbracket_{w ; p} .
\end{gathered}
$$

Since $\llbracket \mathcal{F}(w, q) \rrbracket_{w ; 0}=I_{0}(q)$ and $\llbracket \mathcal{F}(w, q) \rrbracket_{w ; 1}=I_{0}(q) J(q)$, (5.25) follows by adding up the last two equations and using (2.4), the second identity in (4.5), and (4.4).

We now complete the proof of Theorem 3. By Proposition 3.1, Lemma 5.2, (5.3), and the definition of $B(q)$ above,

$$
\mathcal{B}_{i}(Q)-\alpha_{i}^{n-2} \frac{\langle\mathbf{a}\rangle}{24} B(q) \in \mathcal{I} \cdot \tilde{\mathbb{Q}}_{i}[\alpha]^{S_{n-1}}[[q]]
$$

\footnotetext{
${ }^{11}$ Because $a_{k} \neq 1$ by assumption.
} 
By Proposition 3.1, Lemma 5.2, (5.4), and (5.24),

$$
\sum_{j=1}^{n} \tilde{\mathcal{B}}_{i j}(Q)+\frac{\langle\mathbf{a}\rangle}{24} \sum_{j=1}^{n}\left(\frac{\alpha_{j}^{n-2}}{\prod_{k \neq j}\left(\alpha_{j}-\alpha_{k}\right)}\left[\left(Q \frac{\mathrm{d} \tilde{\mathbb{B}}}{\mathrm{d} Q}(q)+B(q)\right)\right] \alpha_{i}^{n-2} \alpha_{j}\right) \in \mathcal{K}_{i}[[q]] .
$$

By the last two equations, (5.22), and (5.23),

$$
\mathcal{B}_{i}(Q)+\sum_{j=1}^{n} \tilde{\mathcal{B}}_{i j}(Q)-\alpha_{i}^{n-2} Q \frac{\mathrm{d} \tilde{B}(q)}{\mathrm{d} Q} \in \mathcal{K}_{i}[[q]] .
$$

This concludes the proof of Theorem 3 ,

\section{REFERENCES}

[ABo] M. Atiyah and R. Bott, The moment map and equivariant cohomology, Topology 23 (1984), 1-28. MR721448 (85e:58041)

[BCOV] M. Bershadsky, S. Cecotti, H. Ooguri, and C. Vafa, Holomorphic anomalies in topological field theories, Nucl. Phys. B405 (1993), 279-304. MR1240687 (94j:81254)

[CaDGP] P. Candelas, X. de la Ossa, P. Green, and L. Parkes, A pair of Calabi-Yau manifolds as an exactly soluble superconformal theory, Nuclear Phys. B359 (1991), 21-74. MR.1115626 (93b:32029)

[ESt] G. Ellingsrud and S. Strömme, Bott's formula and enumerative geometry, J. Amer. Math. Soc. 9 (1996), 175-193. MR.1317230 (96j:14039)

[Gi] A. Givental, Equivariant Gromov-Witten Invariants, IMRN No.13 (1996), 613-663. MR.1408320 (97e:14015)

[KlPa] A. Klemm and R. Pandharipande, Enumerative geometry of Calabi-Yau 4-Folds, Comm. Math. Phys. 281 (2008), no. 3, 621-653. MR2415462 (2009e:14089)

[LiZ] J. Li and A. Zinger, On the genus-one Gromov-Witten invariants of complete intersections, J. Differential Geom. Volume 82, Number 3 (2009), 641-690. MR2534991

[LLY] B. Lian, K. Liu, and S.T. Yau, Mirror Principle I, Asian J. of Math. 1, no. 4 (1997), 729-763. MR.1621573 (99e:14062)

[MirSym] K. Hori, S. Katz, A. Klemm, R. Pandharipande, R. Thomas, C. Vafa, R. Vakil, and E. Zaslow, Mirror Symmetry, Clay Math. Inst., Amer. Math. Soc., 2003. MR2003030 (2004g:14042)

[PaZ] R. Pandharipande and A. Zinger, Enumerative geometry of Calabi-Yau 5-folds, New Developments in Algebraic Geometry, Integrable Systems and Mirror Symmetry, ASPM 59 (2010), 239-288. MR2683212

[PoZ] A. Popa and A. Zinger, Mirror Symmetry for Closed, Open, and Unoriented GromovWitten Invariants, math/1010.1946v3.

[Sc] E. Scheidegger, unpublished note, 4 pages.

[VaZ] R. Vakil and A. Zinger, A desingularization of the main component of the moduli space of genus-one stable maps into $\mathbb{P}^{n}$, Geom. and Top. 12 (2008), no. 1, 1-95. MR2377245 (2009b:14023)

[ZaZ] D. Zagier and A. Zinger, Some properties of hypergeometric series associated with mirror symmetry, Modular Forms and String Duality, 163-177, Fields Inst. Commun. 54, AMS 2008. MR2454324 (2010b:33015)

[Z1] A. Zinger, Reduced genus-one Gromov-Witten invariants, J. Differential Geom. Volume 83, Number 2 (2009), 407-460. MR2577474 (2011d:53227)

[Z2] A. Zinger, On the structure of certain natural cones over moduli spaces of genus-one holomorphic maps, Adv. Math. 214 (2007) 878-933. MR2349722 (2008k:14107)

[Z3] A. Zinger, Genus-zero two-point hyperplane integrals in the Gromov-Witten theory, Comm. Anal. Geom. 17 (2010), no. 5, 1-45. MR2643736 (2011c:14148)

[Z4] A. Zinger, Standard vs. reduced genus-one Gromov-Witten invariants, Geom. and Top. 12 (2008), no. 2, 1203-1241. MR2403808(2009i:14085) 
[Z5] A. Zinger, The reduced genus 1 Gromov-Witten invariants of Calabi-Yau hypersurfaces, J. Amer. Math. Soc. 22 (2009), no. 3, 691-737. MR2505298 (2010c:14066)

[Z6] A. Zinger, The genus 0 Gromov-Witten invariants of projective complete intersections, math/1106.1633v2.

Department of Mathematics, SUNy Stony Brook, Stony Brook, New York 11794-3651

Current address: Department of Mathematics, Rutgers University-Hill Center for the Mathematical Sciences, 110 Frelinghuysen Rd., Piscataway, New Jersey 08854-8019

E-mail address: alexandra@math.rutgers.edu 